TAUP- 2152-94

August 18, 2019

\title{
Folds in 2D String Theories
}

\author{
O. Ganor, J. Sonnenschein and S. Yankielowicz t \\ School of Physics and Astronomy \\ Beverly and Raymond Sacler \\ Department of Exact Sciences \\ Tel-Aviv University \\ Ramat Aviv Tel-Aviv, 69987, Israel
}

\begin{abstract}
We study maps from a 2D world-sheet to a $2 \mathrm{D}$ target space which include folds. The geometry of folds is discussed and a metric on the space of folded maps is written down. We show that the latter is not invariant under area preserving diffeomorphisms of the target space. The contribution to the partition function of maps associated with a given fold configuration is computed. We derive a description of folds in terms of Feynman diagrams. A scheme to sum up the contributions of folds to the partition function in a special case is suggested and is shown to be related to the Baxter-Wu lattice model. An interpretation of folds as trajectories of particles in the adjoint representation of $S U(N)$ gauge group in the large $N$ limit which interact in an unusual way with the gauge fields is discussed.
\end{abstract}

\footnotetext{
${ }^{1}$ Work supported in part by the US-Israel Binational Science Foundation and the Israel Academy of Sciences.
} 


\section{Introduction}

We study the space of maps, including folds, of a two dimensional NambuGoto (NG) string theory. The recent renewed interest in the NG theory was triggered by the discovery of stringy behavior of $S U(N)$ Yang-Mills theory in the large $N$ limit [1, 2, 3]. The latter theory was shown to be equivalent to a $2 \mathrm{D}$ string theory. This equivalence is based on identifying the string coupling constant with $\frac{1}{N}$ and the string tension with $\frac{1}{2} \tilde{g}^{2} N$ where $\tilde{g}$ is the gauge coupling. The asymptotic expansion of the $Y M_{2}$ partition function was recast in terms of a sum of maps from the compact Riemann surface world-sheet to a 2D target space [1, 2, 3]. Point singularities of these maps, like branch points played an important role in the correspondence between the 2D string theory and the $Y M_{2}$ theory. However, line singularities, which will be shown to correspond to folds, had to be excluded by hand. It is the absence of folds which accounted for the fact that pure 2D $Y M_{2}$ theory does not include propagating particles. Using the same logic one is led to consider

folds while searching for the stringy picture of $Q C D_{2}$. Indeed an analysis of folded maps both classically and in a framework of canonical quantization revealed a behavior of particle trajectories [4]. Following the work of D.

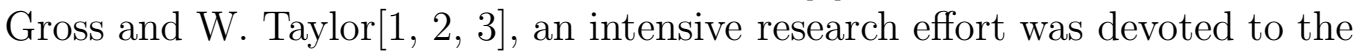
stringy behavior of two dimensional YM theory [5, 6]

A natural candidate for the stringy YM action would have been the Nambu Goto action. However, for a two dimensional target space the NG action is equivalent to a sum of a topological number, the winding number, and a term that counts the area enclosed by folds. Hence, the NG theory cannot be associated, without further alteration, with stringy $Y M_{2}$. Up to date the only consistent method to quantize the NG theory was to translate the classically equivalent formulation of Polyakov [7] and quantize the latter. For a two dimensional target space this is the Liouville continuum formulation of the non-critical string theory. As was discovered in the work of Gross and Taylor [2, 3] singular maps play a major role in performing the sum of maps that corresponds to the partition function of the $Y M_{2}$ theory. The action of Polyakov[]] is not an adequate scheme to discuss these types of maps. In fact, it is easy to realize that gauge fixing the world-sheet reparametrization in the conformal gauge (or the world-sheet light-cone gauge) forbids altogether folds and certain point singularities.

Another path which was followed in the search for a first quantized ac- 
tion of stringy $Y M_{2}$ was that of topological string models. A positive sign in this direction was the fact that the torus YM chiral partition function was reproduces in terms of a summation over holomorphic maps from a toroidal worldsheet $[8]$. Indeed proposals for a topological sigma model action of stringy YM theory were recently written down [9, 10].

Our motivation to study folded maps was thus two folded: (i) Folds as a possible framework to describe particle trajectories in the context of stringy $Q C D_{2}$. (ii) The contribution of folded maps to the functional integral quantization of the NG theory.

In the present work we study maps from a compact Riemann surface to a compact Riemann surface, the world-sheet and the target space respectively. The main idea is to explore the space of maps including folded maps. Unfolded maps are characterized by the numbers of sheets with the same and opposite orientations as that of the target space, and by their singular points. The latter include branch points, tubes that connect sheets of the cover with the same and opposite orientations, and contracted handles [3]. Folds are boundaries of "smooth regions" which are covered with sheets of both orientabilities. Along these boundaries, the sheets of two regions are continued with a (possibly) non-trivial permutation among the sheets, and also, two other sheets of opposite orientability and of the same side of the fold are joined by the fold. Folds are thus characterized by their trajectories on the target space, and by certain types of singular points on them. An algebraic as well as pictorial description of the latter is presented in our work.

An essential step in the analysis of folds is to write down the full measure of the generating functional which includes folds. The measure is induced from the metric on the space of maps that is itself naturally induced from the maps. We found out that the metric on the space of maps includes in addition to the metric of unfolded maps two additional terms, one which is expressed in terms of an integral over the proper length of the fold, and another one which involves the singular points on the fold. Due to these two additional terms the corresponding measure ceases to be invariant under area preserving diffeomorphisms of the target space. Note that once we incorporate folds the correspondence to a $Y M_{2}$ theory is lost. Thus, non-invariance does not create any difficulty. Moreover, if folds are associated with particles interacting with the gauge fields, there is no reason to anticipate such an invariance. It is emphasized in our work that the measure of the full NG theory has many 
undetermined parameters and thus it defines in fact a large family of string models.

The nature of folds as particle trajectories is demonstrated in the computation of the contribution to the partition function of maps from a sphere to a sphere with winding number one and a single fold. This result is then generalized to the case where the bounded region is covered by an arbitrary number of covers from both orientabilities. We develop a set of Feynman diagram rules for maps that include several, possibly intersecting folds. These include expressions for fold propagators, vertices of intersecting folds, the factors which are associated with smooth regions etc. The implementation of these Feynman diagram method to determine the partition function seems to be a difficult task. The expansion parameter is discussed. For the case of a fold enclosing one cover of each orientability, we translate the problem to that of an Ising like model perturbed by triple interaction terms. The interpretation of folds as particles was further clarified by applying the techniques developed in [3] to handle Wilson lines. Using the "generalized Frobenious characters" we found that the operator which is associated with a fold could be understood as a trajectory of a particle in the adjoint representation with an unusual interaction with the gauge fields.

The paper is organized as follows. In section (2) we present the target space picture of the $2 D$ string theory via the NG formulation. Folds and point singularities of these maps are described algebraically as well as pictorially. In particular we analyze singular points on folds, branch points and "quartic points". Section (3) is devoted to the measure on the space of maps. We start with the measure of unfolded maps, emphasizing that there are many undetermined parameters in the model and hence it defines in fact a large family of string theories. We then write down a metric on the space of folded maps and show that it introduces non-invariance under target space area preserving diffeomorphisms. World sheet reparametrization is discussed. We show that standard gauges are not adequate.

In section (4) a computation of the contribution of a fold to the partition function is presented. This is done at first for the simplest fold and then generalized to the case of an arbitrary number of covers via the introduction and calculation of the "fold transition matrix". We discuss the intersection of folds, introduce the notion of "quartic points" and write down a set of Feynman diagrams. The summation over fold configurations is the topic of section (5). We introduce a scheme for performing this sum which is 
related, at least for the case of low cover numbers, to an Ising-like model with triple interactions (known as the Baxter-Wu model) [12. Section (6) is devoted to the interpretation of the folds as particle trajectories and the correspondence to the string description of $Y M_{2}$ theory [2, 3]. We use the tools developed for evaluating Wilson lines to handle folds. It is shown that folds behave like trajectories of particles in the adjoint representation of a $S U(N)$ gauge group in the large $N$ limit, and that singular points on folds introduce peculiar interaction with the jump in $\operatorname{Tr}\left(F^{2}\right)$ along the fold. In section (7) we summarize, present some conjectures and state certain open questions. Explicit calculations of the fold matrix are presented in the appendices. In Appendix A the fold matrix for a fold with no branch points is computed. Appendix B is devoted to the calculation of the eigenvalues of the "fold-matrix " and the "restricted fold matrix".

\section{The target space picture}

Let $\xi^{\alpha}, \alpha=1,2$ denote the coordinates on $\Sigma_{g}$, a genus $g$ world sheet, and $X^{\mu}\left(\xi^{\alpha}\right)$ denote a map from $\Sigma_{g}$ to $\mathcal{M}$, a $D=2$ dimensional target space. The most natural framework to study these maps is the Nambu-Goto (NG) string theory for which the partition function is given by

$$
\mathcal{Z}_{\mathcal{M}}=\sum_{g} \lambda_{s t}^{2 g-2} \int \mathcal{D} X e^{\int d^{2} \xi \sqrt{\operatorname{det}\left(g_{\alpha \beta}\right)}}
$$

where

$$
g_{\alpha \beta}=G_{\mu \nu}(X) \partial_{\alpha} X^{\mu} \partial_{\beta} X^{\nu}
$$

is the metric on the world sheet induced from the target space metric, $G_{\mu \nu}(X)$ is the target space metric and $\lambda_{s t}$ is the string coupling constant. In fact for target spaces of dimension $D=2$ the $\mathrm{NG}$ action takes a simpler form

$$
S=\int d^{2} \xi \sqrt{\operatorname{det}\left(G_{\mu \nu}\right)}\left|\operatorname{det}\left(\frac{\partial X^{\mu}}{\partial \xi^{\alpha}}\right)\right| .
$$

Classically the NG action is equivalent to the action written in the formulation of Polyakov [7]. So far the only consistent way to quantize the continuum 2D string theory has been using the latter approach. As will be shown later, Polyakov's string is not an adequate arena to analyze folds. It 
is thus the idea of the present work to treat the quantum theory in the NG framework.

It is well known that the action and measure are invariant under area preserving diffeomorphisms (APD). The invariance of the measure $\mathcal{D} X$ under $X(\xi) \rightarrow Y(X(\xi))$ where $Y(X)$ is a coordinate transformation on the target space follows because the change of the measure obeys

$$
\frac{\mathcal{D} X}{\mathcal{D} Y}=\prod_{\xi} \operatorname{det}\left(\frac{\partial Y^{\mu}}{\partial X^{\nu}}\right)
$$

where for APD the determinants are 1. The action (3) is obviously also unmodified by these diffeomorphisms. However, as will be shown below, this does not mean that the NG theory itself has the APD symmetry.

According to whether the determinant of the Jacobian matrix

$$
J_{\alpha}^{\mu}=\frac{\partial X^{\mu}}{\partial \xi^{\alpha}}
$$

vanishes or not, we classify the maps $X^{\mu}\left(\xi^{\alpha}\right)$ into two types. Unfolded maps correspond to $\operatorname{det}(J) \neq 0$ everywhere. Folds, as will be clarified in the following section, are curves on the target space on which $\operatorname{det}(J)=0$. It is only when we restrict to the space of unfolded maps that the theory becomes invariant under this symmetry. In section (3.2) it is shown that folds turn the measure $\mathcal{D} X$ into a singular one and in order to correct its singularity we have to break APD invariance.

Let us proceed with a short reminder on unfolded maps. These maps are taken to be locally covering maps of $\mathcal{M}$ apart from a finite set of singular points. They are characterized by the number of sheets with the same orientation as that of $\mathcal{M}$ and by the number of sheets of opposite orientation. In addition the maps are classified according to their singular points. The singularities include branch points, contracted tubes that connect sheets of the cover with the same and opposite orientations, and contracted handles[3]. It was shown by D. Gross and W. Taylor [2, 3] that the $S U(N) Y M_{2}$ theory at $N \rightarrow \infty$ is reproduced by summing over unfolded maps which include branch points, contracted tubes, contracted handles as well as $2 g-2$ " $\Omega$-points". 


\subsection{Geometry of the Folded Maps}

Let us start with a pictorial description of certain geometrical structures that arise with folded maps. The general form in target space of a map $X: \Sigma_{g} \rightarrow \mathcal{M}$ is of regions with no folds that are bounded by folds (Fig.1). The folds are thus considered as boundaries of "smooth regions". In each such region there might be a multiple cover with sheets of both orientabilities. For instance in Fig.2, the region under the "pocket" has three sheets one with a $(-)$ orientability and the other two with a $(+)$ orientability. The region under the "smashed handle" of Fig.3 has the same cover-structure. Along the folds, the sheets of two regions are continued with a (possibly) non-trivial permutation among the sheets, and also, two sheets of opposite orientability and of the same side of the fold are joined by the fold. Thus, for each fold one side of it has two more sheets than the other - one sheet more for each orientability.

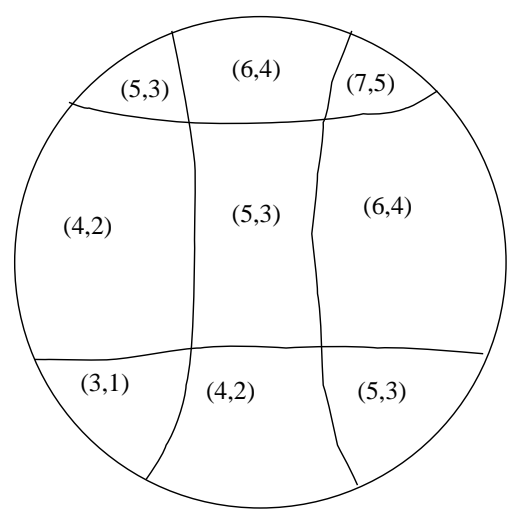

Fig. 1: A part of a target space that is covered by a map with folds. The areas $(m, n)$ represent regions with $m$ sheets of $(+)$ orientation and $n$ sheets of $(-)$ orientation. 

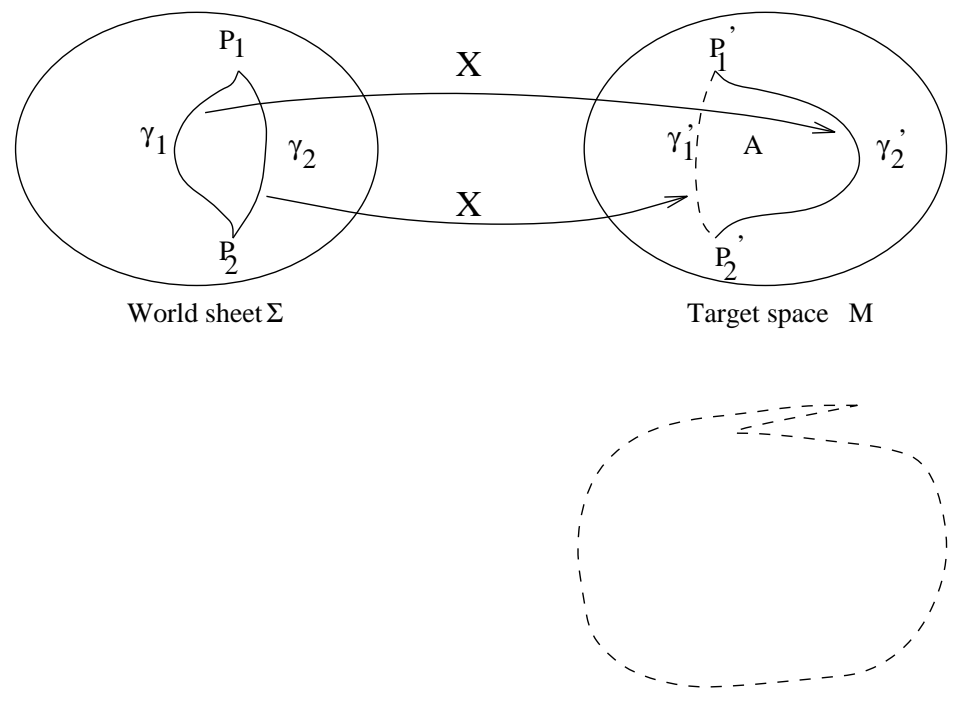

Side-view

Fig. 2: A "pocket" is formed when an area $A$ in target space is a 3-sheet cover inside a 1-sheet cover $M-A$. The boundary curve has two singular points $P_{1}, P_{2}$ where the joining of the sheets changes. 


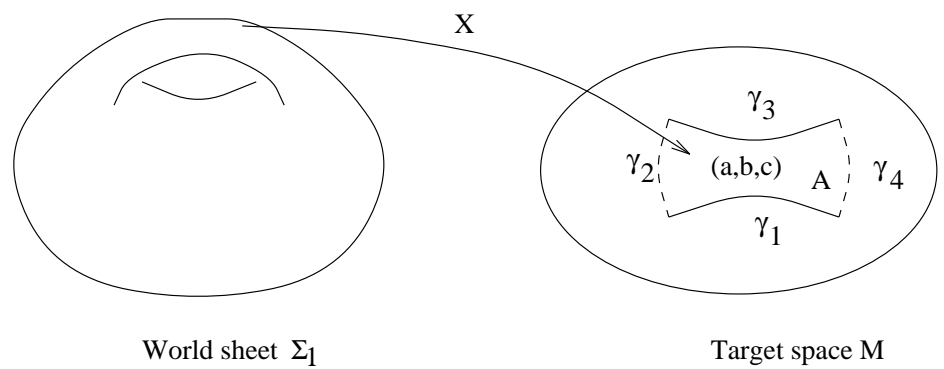

Fig. 3: A "smashed handle" is formed when a 3-sheet area $A \subset M$ is bounded by a curve with 4 singular points. Along $\gamma_{1}, \gamma_{3}-$ ' $a$ ' and ' $b$ ' are joined and 'c' continues smoothly to $M-A$. Along $\gamma_{2}, \gamma_{4}-$ 'c' and ' $b$ ' are joined and ' $a$ ' continues smoothly to the outside.

A fold is a curve on which $\operatorname{det}\left(J_{\alpha}^{\mu}\right)=0$. Along the folds there might be several singular points where the joining of the sheets changes as can be seen in Fig.2. It might seem that such points are singular also geometrically, that is they are points at which the direction of the tangent along the fold is discontinuous. It is shown in what follows that generically this is not the case . Namely, we will see that (generically) points with a discontinuous tangent are points where the Jacobian matrix $J_{\alpha}^{\mu}$ vanishes altogether, whereas along folds only the determinant $\operatorname{det}\left(J_{\alpha}^{\mu}\right)$ vanishes, and at singular points along a fold the determinant has in some sense (to be made precise later on) a double zero.

Let us analyze algebraically the possible singular points of the general map

$$
\begin{aligned}
& X_{1}=f\left(\xi_{1}, \xi_{2}\right) \\
& X_{2}=g\left(\xi_{1}, \xi_{2}\right)
\end{aligned}
$$

at the vicinity of the origin $\xi_{1}=\xi_{2}=0$. Expanding in a Taylor series we get after performing a local world sheet reparametrization the generic map

$$
\begin{aligned}
& X_{1}=\xi_{1} \\
& X_{2}=\xi_{2}
\end{aligned}
$$


which is a point inside a smooth region.

The other cases happen when some of the derivatives of $f$ or $g$ vanish. In each case we expand in leading order in $\xi_{1}, \xi_{2}$, performing a world-sheet reparametrization and a target space Lorentz rotation and a parity transformation if necessary. We will restrict ourselves to situations where up to two conditions are imposed on the derivatives of $f$ and $g$ because a generic map $X^{\mu}\left(\xi^{\alpha}\right)$ has no points on the world-sheet where three conditions are satisfied (three equations in two variables). In what follows $A, B, C, \ldots$ are parameters. The various possible cases are:

1. A simple point on a fold

This is the case when $\operatorname{det}\left(J_{\alpha}^{\mu}\right)=0$. We can bring the map to a form

$$
\begin{aligned}
& X_{1}=\xi_{1}^{2}+A \xi_{2}+\cdots \\
& X_{2}=\xi_{2}
\end{aligned}
$$

where $X_{1}$ has no linear term in $\xi_{1}$. This map has a fold in the vicinity of $(0,0)$ on the curve $X_{1}=A X_{2}$. It describes two sheets below it and no sheets above it.

2. A singular point on a fold

We can supplement the condition $\operatorname{det}\left(J_{\alpha}^{\mu}\right)=0$ with a further condition as follows. If $J_{\alpha}^{\mu} \neq 0$ as a matrix, then there is a direction $a^{\alpha} \partial_{\alpha}$ in the tangent space to the world-sheet at the origin $T \Sigma_{g}$ such that

$$
a^{\alpha} \partial_{\alpha} X^{\mu}=0, \quad \mu=1,2
$$

We demand that

$$
a^{\beta} \partial_{\beta} \operatorname{det}\left(J_{\alpha}^{\mu}\right)=0
$$

as well. For a generic point on a fold the direction $a^{\alpha} \partial_{\alpha}$ is different from the fold direction (the direction in which $\operatorname{det}\left(J_{\alpha}^{\mu}\right)$ remains zero), but for the singular point that we discuss now the direction $a^{\alpha} \partial_{\alpha}$ coincides with the direction of the fold. In the vicinity of such a point the map looks like

$$
\begin{aligned}
& X_{1}=\xi_{1}^{3}-A \xi_{1} \xi_{2}+B \xi_{1}^{2} \xi_{2}+C \xi_{2}+D \xi_{2}^{2}+\cdots \\
& X_{2}=\xi_{2}
\end{aligned}
$$


The qualitative analysis is not affected by taking $B=C=D=0$ for simplicity. In that case the equation of the fold is

$$
3 \xi_{1}^{2}-A \xi_{2}=0
$$

The direction $a^{\alpha} \partial_{\alpha}$ is $\partial_{1}$ which is also the direction of the fold (in the world sheet).

In target space the fold looks like

$$
X_{1}^{2}=4\left(\frac{A X_{2}}{3}\right)^{3}+O\left(X_{2}^{\frac{7}{2}}\right)
$$

and we see that it has a smooth tangent vector (although it has an infinite curvature at the origin).

Fixing $\xi_{2}$ and varying $\xi_{1}$ we obtain $X_{1}$ as a cubic function of $\xi_{1}$. For $\xi_{2}<0$ the function is monotonous, and thus there is one sheet in the target space below the curve $X_{1}^{2}=\frac{4}{3} A^{3} X_{2}^{3}$. For $\xi_{2}>0$ the cubic function is not monotonous any more and passes through each point above the curve $X_{1}^{2}=4\left(\frac{A X_{2}}{3}\right)^{3}$ thrice. (See Fig.4).

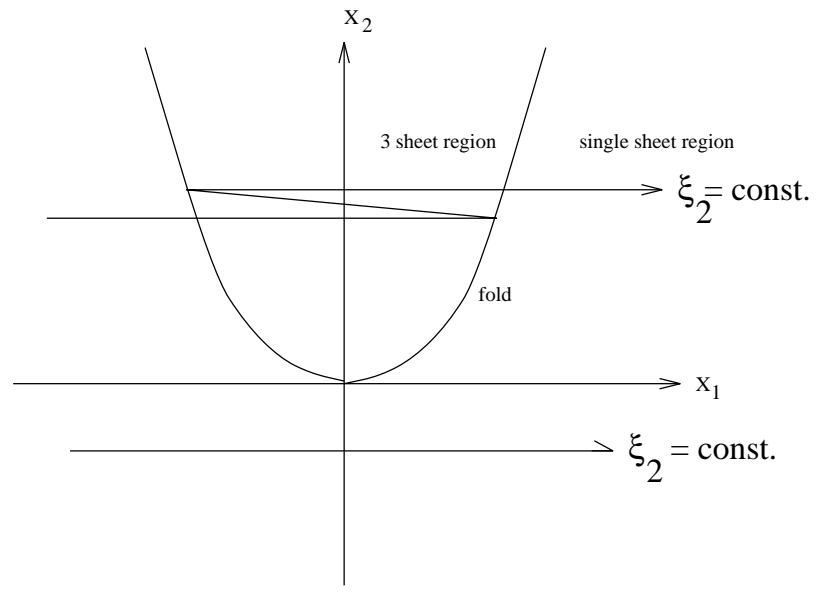

Fig. 4: The map of equation (8) for $B=C=D=0$ creates a fold with a singular point at the origin. (The $\xi_{2}=$ const. line was split for the sake of visibility. It should have been overlapping.) 
3. A branch point

When the Jacobian matrix vanishes two cases can arise. They both correspond to maps of the form

$$
\begin{aligned}
& X_{1}=A \xi_{1}^{2}+2 B \xi_{1} \xi_{2}+C \xi_{2}^{2} \\
& X_{2}=D \xi_{1}^{2}+2 E \xi_{1} \xi_{2}+F \xi_{2}^{2}
\end{aligned}
$$

substituting

$$
\begin{aligned}
& \xi_{1}=r \cos t \\
& \xi_{2}=r \sin t
\end{aligned}
$$

for an infinitesimal $r$ we see that a circle around $(0,0)$ in the world sheet goes to an ellipse of winding number 2 on the target space. When the origin $(0,0)$ is inside the ellipse, $(0,0)$ is a branch point. This happens when

$\Delta=A^{2} F^{2}+D^{2} C^{2}-4 B E D C-4 B E A F+4 E^{2} A C+4 B^{2} D F-2 A C D F<0$

Such a branch point is depicted in Fig.5. 


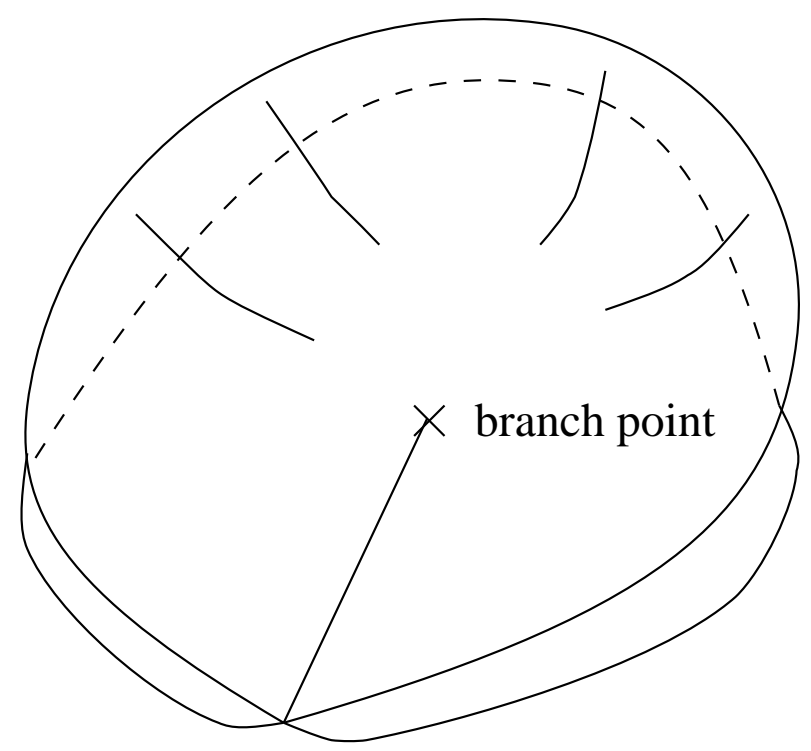

Fig. 5: A branch point

4. A quartic point

The other case corresponds to a map of the form

$$
\begin{aligned}
& X_{1}=A \xi_{1}^{2}+2 B \xi_{1} \xi_{2}+C \xi_{2}^{2} \\
& X_{2}=D \xi_{1}^{2}+2 E \xi_{1} \xi_{2}+F \xi_{2}^{2}
\end{aligned}
$$

with

$$
\Delta=A^{2} F^{2}+D^{2} C^{2}-4 B E D C-4 B E A F+4 E^{2} A C+4 B^{2} D F-2 A C D F>0
$$

We see that it corresponds to two double folds that meet at an angle. They separate a region of four sheets from a region of no sheets. Adding cubic terms to the functions $X^{\mu}\left(\xi^{\alpha}\right)$ we obtain a splitting of the double folds and get four folds that meet at a "quartic" point (See Fig.6). 


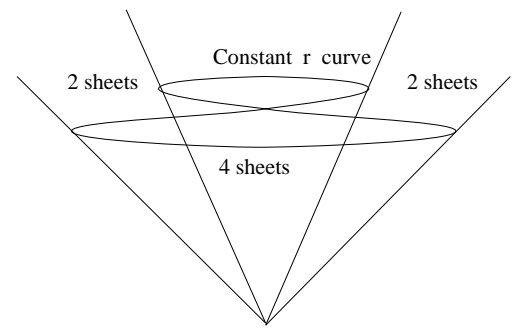

Fig. 6: A quartic singular point (schematically). (The $r=$ const. curve was perturbed for the sake of clearer visibility. It should have been overlapping.)

The preceding discussion can obviously be generalized to maps which are higher order polynomials. This will introduce higher order branch points. Nevertheless, as was mentioned above, classically, generic maps do not have those higher order branch points.

\section{The Measure on the space of Maps}

With the definitions of folds and singular points on them at hand, our next object of interest is the measure on the entire space of maps $X^{\mu}\left(\xi^{\alpha}\right)$ from $\Sigma_{g}$ to $\mathcal{M}$. It will be shown that in order to incorporate the geometrical configurations such as the location of the folds and singular points one cannot use the metric of the subspace of unfolded maps. Instead one has to add to the latter two additional terms. We start our discussion with the measure of the unfolded maps, introduce the necessary modifications to incorporate folds and the singular points on them, and analyze the invariance under APD.

\subsection{The Measure on the Space of Unfolded Maps}

Unfolded maps can be classified according to their world-sheet independent properties namely, the winding number and the location of branch-points. On the other hand, the measure in the Nambu-Goto formalism is proportional to $\prod_{\xi} d X(\xi)$ modulo world-sheet diffeomorphisms. It is specified by specifying 
the corresponding metric

$$
\|\delta X\|^{2}=\int G_{\mu \nu} \delta X^{\mu} \delta X^{\nu} \sqrt{\operatorname{det}\left(g_{\alpha \beta}\right)} d^{2} \xi
$$

where $g_{\alpha \beta}$ is given in (2). The contributions of the singular points of unfolded maps to the measure take the form of $\lambda d A$ where $d A$ is an infinitesimal target space area in which the singular point is located and the $\lambda$-s are parameters of dimension $1 / A$ that specify the type of the singular point, namely $\lambda_{b r}$ for a branch point and (for the case of the string theory of [2] [3]) $\lambda_{c t+}, \lambda_{c t-}$ and

$\lambda_{c h}$ for connecting tubes of the same and opposite orientation and collapsed handles respectively. This is not a consequence of (11) but has to be added "by hand", as will be discussed in more detail in the next subsection.

Apriori we do not see any reason why those parameters have to be equal. The latter case was found to be necessary for a string theory which corresponds to the large $N$ limit of the $S U(N) Q C D_{2}$ theory. It seems to us that the $\lambda$ parameters cannot be determined from the NG measure.

\subsection{The Induced Measure on Folds}

What happens when we perturb a map $X(\xi)$ with no folds with a small perturbation $\delta X(\xi)$ so that the map $X+\delta X$ has an infinitesimal fold? Although $\delta X$ is small, the derivative $\nabla \delta X$ must be non-infinitesimal in order to create a fold since we can reparametrize $\xi$ so that $\nabla X$ will be no-where zero. Although the measure $\|\delta X\|$ can be small, we will refer to maps with folds and maps with no folds as belonging to different "sectors".

The measure on the space of maps, which is specified by the metric (11), is singular at maps with folds. This happens because the metric (11) is not strictly positive definite at maps with folds. At folds $\operatorname{det}\left(g_{\alpha \beta}\right)=0$ and thus $\delta X(\xi)$ for $\xi$ on a fold is a zero mode of the metric. Thus, the measure would be identically zero for maps with folds.

Geometrically, a folded map is specified by the location of its folds and singular points. Thus it seems natural that the induced measure on maps modulo world-sheet diffeomorphisms will be the induced measure on the subset of infinitesimal changes $\delta X(\xi)$ for $\xi$ at folds and singular points. Indeed, if we look at a map $X(\xi)+\delta X(\xi)$ at a point $\xi_{0}$ on a fold, then to first order in $\delta X$ the point $X\left(\xi_{0}\right)+\delta X\left(\xi_{0}\right)$ is on a fold of the new map. In other words 
the folds and singular points of the map $X(\xi)+\delta X(\xi)$ are the locus of the points $X(\xi)+\delta X(\xi)+O\left(\delta X^{2}\right)$ for $\xi$ at a fold of $X(\xi)$.

Let a fold be parametrized in the world-sheet as $\xi^{\alpha}=\xi^{\alpha}(t)$ where $t$ is a real parameter say in $[0,2 \pi)$. In the target space, the fold is parametrized as $X^{\mu}=X^{\mu}(\xi(t))$. In order to have a well defined measure for maps with folds we have to correct the metric (11) and add terms on folds. In order to keep the world-sheet diffeomorphism invariance we have to keep the invariance of the added term under $t \rightarrow \tau(t)$. The term must thus be of the form

$$
\int G_{\sigma \tau} \delta X^{\sigma} \delta X^{\tau} \sqrt{G_{\mu \nu} \frac{d X^{\mu}(\xi(t))}{d t} \frac{d X^{\nu}(\xi(t))}{d t}} d t
$$

The integral is thus over the proper length of the fold. For isolated singular points (including singular points on a fold) we have to add

$$
\sum_{j} G_{\mu \nu} \delta X^{\mu}\left(\xi_{j}\right) \delta X^{\nu}\left(\xi_{j}\right)
$$

where $\xi_{j}$ is the location of the $j$-th singular point. The corrected measure is thus

$$
\begin{aligned}
\|\delta X\|^{2} & =\int G_{\mu \nu} \delta X^{\mu} \delta X^{\nu} \sqrt{\operatorname{det}\left(g_{\alpha \beta}\right)} d^{2} \xi \\
& +a \int G_{\sigma \tau} \delta X^{\sigma} \delta X^{\tau} \sqrt{G_{\mu \nu} \frac{d X^{\mu}(\xi(t))}{d t} \frac{d X^{\nu}(\xi(t))}{d t}} d t \\
& +b \sum_{j} G_{\mu \nu} \delta X^{\mu}\left(\xi_{j}\right) \delta X^{\nu}\left(\xi_{j}\right)
\end{aligned}
$$

where $a$ and $b$ are arbitrary constants.

In principle we could choose instead of the $b$-term a more complicated term with a factor that depends on the distances between the singular points and the lengths of the arcs of a fold between consecutive singular points on it. However, (12) is the only choice that is local in $X(\xi)$. The $a$-term is actually the measure for a relativistic point particle in $2 \mathrm{D}$.

To summarize, we have argued that the measure in target space for this topological sector of maps with a single connected fold (which we assume to be homotopic to a circle) and $k$ singular points can be taken to be proportional to:

$$
\frac{\prod_{t}\left(d X^{1}(\xi(t)) d X^{2}(\xi(t))\right)}{\operatorname{Diff}_{1}(t \mapsto \tau(t))} \prod_{i=1}^{k}\left(\frac{d l}{d t_{i}} d t_{i}\right)
$$


where

$$
\frac{d l}{d t}=\sqrt{G_{\mu \nu} \frac{d X^{\mu}(\xi(t))}{d t} \frac{d X^{\nu}(\xi(t))}{d t}}
$$

and $\operatorname{Diff}_{1}(t \rightarrow \tau(t))$ means that we have to gauge fix this one-dimensional group of diffeomorphisms along the fold. The first term in (13) is exactly the "Nambu-Goto" measure for a bosonic point-particle.

The geometrical meaning of $(13)$ is as follows. We know that the full NG measure is

$$
\frac{\prod_{\xi}\left(d X^{1}(\xi) d X^{2}(\xi)\right)}{\operatorname{Diff}_{2}(\xi \mapsto \eta(\xi)) .}
$$

Because of the diffeomorphism invariance any infinitesimal change $d X^{\mu}(\xi)$ for a $\xi$ that is not on the fold (i.e. not on $\xi(t)$ ) can be gauged away by a world-sheet coordinate transformation from Diff 2 . The only changes $d X^{\mu}(\xi)$ that cannot be gauged away are those that deform the fold in target-space, i.e. those for which $\xi=\xi(t)$ for some $t$. For these values of $\xi$ the world sheet diffeomorphism group is reduced to one parameter - that is, only a change $d X^{\mu}$ that is parallel to the tangent to the fold at that point, can be eliminated by a gauge transformation. At the special points $t_{i}$ any infinitesimal translation of the singular point gives a different target space picture, and thus cannot be absorbed by a world-sheet diffeomorphism. Let us decompose

$$
d X^{1}\left(\xi\left(t_{i}\right)\right) d X^{2}\left(\xi\left(t_{i}\right)\right)=d l_{i} d y_{i}
$$

where $d l_{i}$ is a translation of the singular point in a direction parallel to the fold, and $d y_{i}$ is a translation perpendicular to the fold (that cannot be removed by a world-sheet diffeomorphism). The part $d y_{i}$ contributes to the measure of the fold (modulo Diff 1 ) while $d l_{i}$ is the measure for the location of the singular point on the fold. We thus obtain (13).

The theory can be rigorously defined on a discrete target space. We will pick a triangulation of the target space and define the discrete configuration space of the theory as all the possible ways to associate two integer covernumbers with each elementary cell of the triangulation, and to specify how the sheets join each other on the edges where two cells touch. In this way we bypass the need for a world-sheet and do not have any reparameterization invariance. This is different from the continuum version in the fact that singular points of all types (branch points, singular points on folds, or spiral points) can exist only on the vertices of the triangulation and folds can exist 
only on the edges. The discrete maps are combinatorial objects. If we restrict the configuration space of all such combinatorial maps, to those that have no spiral points and no more than one fold on every edge, we will get a lattice version of the measure (13).

\subsection{Invariance under area preserving diffeomorphisms}

As was mentioned in the previous section, the NG action is invariant under APD of the target-space. The measure $\mathcal{D} X$ seems also invariant since $\operatorname{det}\left(g_{\alpha \beta}\right)$ in (11) is APD invariant and the determinant of the metric in the space of maps is thus APD invariant. However, it is easy to realize that because of the $a$-term and the "proper" length along the fold, (12) is not APD invariant.

The term $\prod_{t}\left(d X^{1}(t) d X^{2}(t)\right)$ which appears in the measure as a consequence of the $a$-term in (12) is invariant under (target-space) APD, however,

the measure has the additional factor of $\frac{d s}{d t}$ where $d s$ is the proper time, and this breaks APD invariance.

It is interesting to note that there exists an area preserving diffeomorphism of the target space that changes the fold $Y^{\mu}(t)$, where $t \in[0,2 \pi)$ is parameterizing the fold, to any other form $Z^{\mu}(t)$ provided it has the same area.

We will show it for infinitesimal changes $\delta Y^{\mu}(t)$ that satisfy

$$
\int \epsilon_{\mu \nu} \delta Y^{\mu}(t) \dot{Y}^{\nu}(t) d t=0
$$

representing the fact that the area is unchanged. We want to extend $\delta Y^{\mu}(t)$ to a function on the whole of target space $u^{\mu}(X)$ such that

$$
\begin{aligned}
& u^{\mu}(Y(t))=\delta Y^{\mu}(t) \\
& \partial_{\mu} u^{\mu}(X)=0
\end{aligned}
$$

The second identity represents the invariance of $\sqrt{g}$. We can seek a solution in the form

$$
u^{\mu}(X)=\epsilon^{\mu \nu} \partial_{\nu} \phi(X)
$$

which is consistent with (17). Clearly $\phi(X)$ can be chosen so as to satisfy the boundary condition on the folds. 


\subsection{World-Sheet Reparametrization Gauge Fixing}

Gauge fixing of the world-sheet diffeomorphisms requires a special care in string theory with folds. This is demonstrated in the use of the conformal gauge. The latter means inserting the delta functions $\prod_{\xi} \delta\left(g_{++}(\xi)\right) \delta\left(g_{--}(\xi)\right)$ together with the corresponding Fadeev-Poppov determinant. However, this procedure is not applicable when the map $X$ has folds. The reason is that on folds the map $X$ is singular, and thus $\operatorname{det}\left(g_{\alpha \beta}\right)=0$. In the conformal gauge this implies that $g_{\alpha \beta}=0$ on folds, and thus, since the perimeter of a fold is greater than zero, the fold must lie at the infinity of the $\xi$ parameter space. Using the world-sheet light-cone gauge is also not adequate since inherently in that gauge $\operatorname{det}\left(g_{\alpha \beta}\right)=1$. One can try to overcome this problem by, for instance, fixing $g_{++}(X)=g_{++}^{0}$ where $g_{++}^{0}$ is some nonzero constant value, (and similarly $g_{--}(X)=g_{--}^{0}$ ), however this will prevent the map $X$ from having branch points at which $g_{\alpha \beta}=0$.

\section{The contribution of folded maps to the par- tition function}

In the following sections we will concentrate on the consequences of the $b$ term in (12). Our approach will be to sort the maps into sectors. Each sector will be specified by the way in which the folds divide the target-space. More precisely, it will be specified by the graph that is formed from the smooth regions (as vertices of the imaginary graph) and the folds (as edges connecting the smooth regions).

We then have to sum over all possible number of branch points inside the smooth regions, and all possibilities of singular points on the folds. The measure for the latter will be given by the $b$-term of (12). The summations are essentially of a combinatorial nature, because we have to consider various ways in which the sheets of the smooth regions join.

After we have done this, we should, in principle, integrate over all the maps of the sector. This involves an integration over the location of the folds and is given by the $a$-term of (12). In contrast to the $b$-term contribution, the $a$-term contribution is of a dynamical nature. It involves integration over self-avoiding random walks.

In section (2.2) we studied the classical possibilities for singular points in 
a map. Our assumption was that the map function $X^{\mu}\left(\xi^{\alpha}\right)$ can be Taylor expanded and that the maps are generic. In particular we saw that the form of singular points on a fold is very restricted and, for example, a singular point on a fold, for a generic map cannot have a branch cut starting from it. "Spiral-points" (as in Fig.7) are excluded.

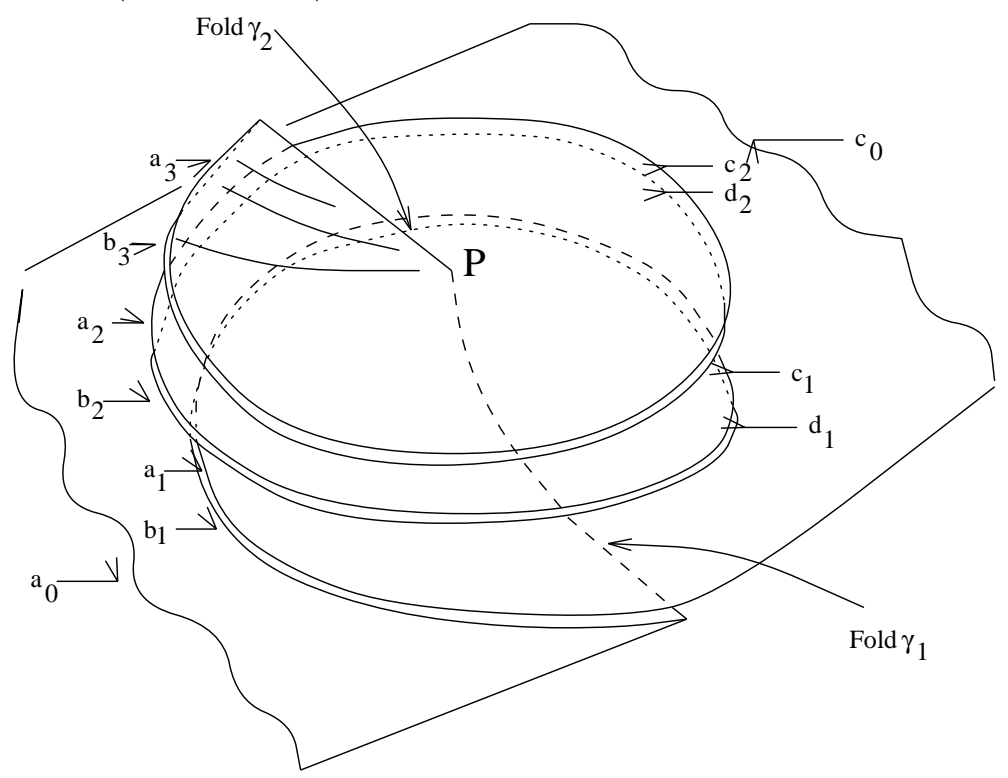

Fig. 7: $P$ is a spiral point of order $2 \frac{1}{2}$. along $\gamma_{1}: a_{0}$ and $b_{1}$ are joined by the fold and the smooth passages are: $a_{1}-c_{0}, a_{2}-c_{1}, a_{3}-c_{2}, b_{2}-d_{1}$, $b_{3}-d_{2}$. Along $\gamma_{2}: a_{3}$ and $b_{3}$ are joined by the fold and the smooth passages are: $a_{0}-c_{0}, a_{1}-c_{1}, a_{2}-c_{2}, b_{1}-d_{1}, b_{2}-d_{2}$. 


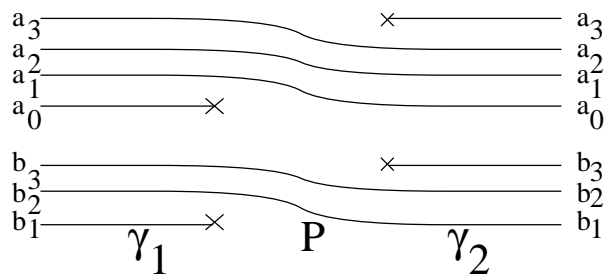

Fig. 8: A graphic representation of the connection of the sheets near $P$.

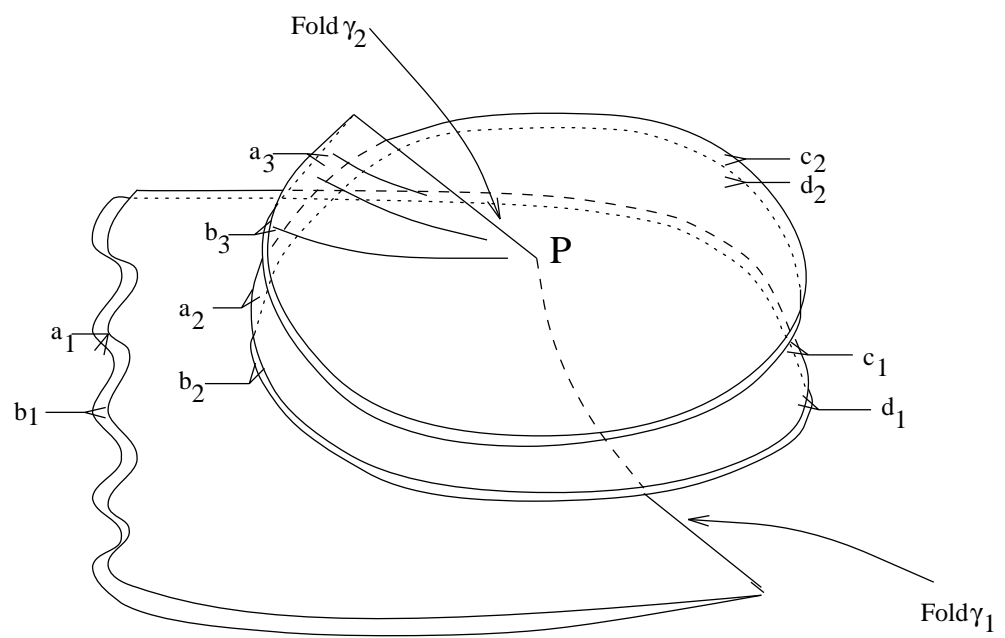

Fig. 9: $P$ is a spiral point of order 2. Along $\gamma_{1}: a_{1}$ and $b_{1}$ are joined by the fold and the smooth passages are: $a_{2}-c_{1}, a_{3}-c_{2}, b_{2}-d_{1}, b_{3}-d_{2}$. Along $\gamma_{2}$ : $a_{3}$ and $b_{3}$ are joined by the fold and the smooth passages are: $a_{1}-c_{1}$, $a_{2}-c_{2}, b_{1}-d_{1}, b_{2}-d_{2}$. 

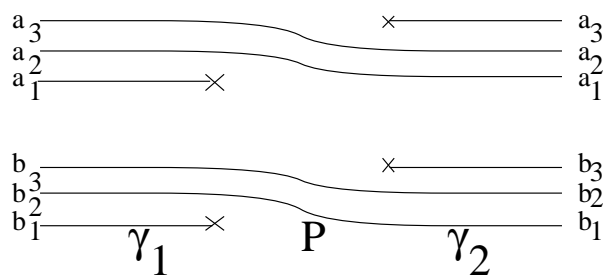

Fig. 10: A graphic representation of the connection of the sheets near P.

These arguments probably do not persist at the quantum level. Renormalizability arguments probably force us to include additional special points like "spiral-points". When we take a cutoff $\Lambda$, of dimension of mass, to regularize the theory we allow a branch point to be close to a fold only by a distance of the order of $\Lambda^{-1}$. All configurations with branch points that are closer than $\Lambda^{-1}$ to a fold should be considered as configurations with branch points on the fold. classically, the "volume" of such configurations (in map space) tends to zero. However, renormalization might give it a nonzero weight. Similarly, infinitesimal contracted handles and connecting tubes which were needed in [3] might arise by a similar mechanism.

We will begin a study of the simple cases that arise, not restricting ourselves to the "classical" configurations. To examine the role played by the folds we start with a simple example of maps from a sphere to a sphere with winding number one and a single fold. Suppose that an area enclosed by the arcs $\gamma_{1}$ and $\gamma_{2}$ in $\Sigma$ is mapped by $X$ to a closed region $A$ in $\mathcal{M}$ in such a way that on $\mathcal{M}$ the region $A$ is a 3 -sheet cover and $\mathcal{M}-A$ is a single-sheet cover. The region $A$ thus forms a pocket (see Fig.2). The two curves $\gamma_{1}, \gamma_{2}$ that mark the folds are mapped into $\gamma_{1}^{\prime}, \gamma_{2}^{\prime}$. If we pick a random labeling of the sheets of $A$ calling them $a, b, c$ then at the boundary $\gamma_{1}^{\prime}$ the sheets $a, b$ are glued together and at $\gamma_{2}^{\prime}$ the sheets $c, b$ are joined together, thus forming a "pocket".

The next topological situation (see Fig.11) we would like to examine is a region $A$ in $\mathcal{M}$ that is a 3 -sheet cover bounded by $k \operatorname{arcs} \gamma_{1}, \gamma_{2}, \ldots, \gamma_{k}$ (we drop the prime from arcs on the target space). We will assume that the 
outside region $\mathcal{M}-A$ is a single sheet. Along each $\gamma_{i}$ two of the three sheets join. We require that at $\gamma_{i}$ and $\gamma_{i+1}$ the joining of the sheets is different $(k+1$ is by definition 1$)$. There are altogether 3 different ways to join two sheets out of three. However two of the sheets have orientability $(+)$, like the outer single sheet and the last sheet has orientability $(-)$. So, if we want an oriented figure, we are allowed to join only a $(+)$ sheet to a $(-)$ sheet. This leaves only two ways to join, and since adjacent $\gamma_{i}$-s must not have the same joining, there are altogether two ways to join the sheets. Since the labeling of the two $(+)$ sheets was arbitrary, there is a symmetry factor of 2 , which cancels out - leaving only one way to join.

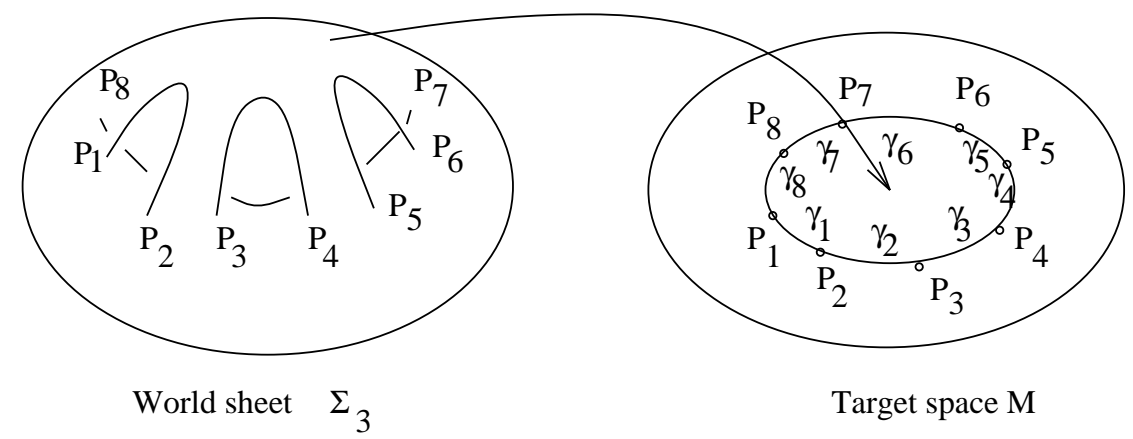

Fig. 11: A map from a world sheet of high genus to a sphere.

The Euler number of $\Sigma, \chi(\Sigma)$, is given by

$$
\chi(\Sigma)=\text { Vertices }+ \text { Faces }- \text { Edges }=4-k
$$

When we consider the partition function of the string we will have to sum over all positions of the $k$ endpoints of the $\gamma_{i}$. Such configurations should thus get a factor of $(\rho l)^{k} / k$ ! where $l$ is the length of the boundary of $A$ and $\rho$ is a parameter of dimension $[\text { Length }]^{-1}$ that is adjoined with singular points.

Clearly $k$ must be even and in order that the world-sheet should be connected we need $k \geq 2$.

Summing over even $k$ one finds 


$$
\begin{aligned}
e^{Z(\mathcal{M})} & =e^{-\mu \cdot \operatorname{Area}(\mathcal{M})-2 \mu \cdot \operatorname{Area}(A)} \sum_{g=0}^{\infty} \lambda_{s t}{ }^{2 g-2} \frac{(\rho l)^{2 g+2}}{(2 g+2) !} \\
& =\frac{1}{2 \lambda_{s t}{ }^{4}} e^{-\mu \cdot \operatorname{Area}(\mathcal{M})-2 \mu \cdot \operatorname{Area}(A)}\left(e^{\lambda_{s t} \rho l}+e^{-\lambda_{s t} \rho l}\right)
\end{aligned}
$$

Notice that we included the term $k=0$ which corresponds to a disconnected world-sheet. We thus calculate $e^{Z(\mathcal{M})}$ rather than $Z(\mathcal{M})$.

Equation (21) suggests a relation between a fold and a trajectory of a point particle. The action of a point particle coupled to a YM $S U(N)$ field is proportional to the length of its trajectory times its mass plus the expectation value of the Wilson loop around its trajectory which is proportional to the area if the trajectory is not self intersecting [11]. According to this interpretation, (21) is a sum of two terms which correspond to a particle of mass $\lambda_{s t} \rho$ and a ghost particle of negative mass $-\lambda_{s t} \rho$. After we perform a Wick rotation we get an imaginary $\rho$ and a real mass $\pm \lambda_{s t}|\rho|$. The negative mass does not create a problem if only the square of the mass appears in final results. However, the interpretation of a massive particle, o is not consistent when we go to higher cover numbers, as we shall discuss later.

\subsection{The Fold Transition Matrix}

In the previous section we considered folded maps based on a simply connected region covered with two sheets of positive orientability $(+)$ and one of negative orientability $(-)$. The region was surrounded, in the rest of the target space, by a single cover $(+)$ sheet. We generalize this simple set of maps to maps with a simply connected region that has $n+1$ sheets of orientability $(+)$ and $m+1$ sheets of orientability $(-)$, whereas the outside region has $n$ sheets of orientability $(+)$ and $m$ sheets of orientability $(-)$.

Again, we pick $k$ singular points along the curve $\gamma, \gamma=\bigcup_{i=1}^{k} \gamma_{k}$ that bounds the region $A$. Along each $\gamma_{i}$ we perform the following operations:

1. join one of the $n+1$ orientability $(+)$ sheets to one of the $m+1$ orientability $(-)$ sheets,

2. connect the $m$ remaining orientability $(-)$ sheets of the inside to the $m$ remaining orientability $(-)$ sheets of the outside in some random permutation, 
3. connect the $n$ remaining orientability $(+)$ sheets of the inside to the $n$ remaining orientability $(+)$ sheets of the outside in some random permutation.

The genus of the obtained figure can be determined as before from Euler's formula. Assuming that the $n+m$ sheets of the outside region are disconnected (in $\mathcal{M}-A$ ) we get:

$$
\begin{aligned}
\text { Vertices } & =(m+n) k+\sum(\text { spiralities }) \\
\text { Faces } & =2 n+2 m+2 \\
\text { Edges } & =(n+m+1) k
\end{aligned}
$$

The term 2 (spiralities) is related to points of the type of Fig.7 or Fig.9 . In the "classical" configurations, as we saw earlier, no such points exist and we obtain that the sum of the Euler numbers is:

$$
\sum_{\text {components }}\left(2-2 g_{i}\right)=2 n+2 m+2-k
$$

We see that each singular point contributes $(-1)$ to the Euler characteristic, and that every cover contributes $(+1)$.

Let $\left(x_{i}, y_{i}\right)$ for $i=1, \ldots, k$ be the sequential numbers of the joined sheets at $\gamma_{i}$. Thus $1 \leq x_{i} \leq m+1$ and $1 \leq y_{i} \leq n+1$. Let $\sigma_{i} \in S_{n+1}$ be defined as follows: $\sigma_{i}(x)$ for $x=1,2, \ldots, n$ will be the sheet number from $1,2, \ldots, n+1$ to which $x$ continues as it crosses the fold, and $\sigma_{i}(n+1)$ is defined to be $x_{i}$. Likewise we define $\tau \in S_{m+1}$ as follows: $\tau_{i}(y)$ for $y=1,2, \ldots, m$ will be the sheet number from $1,2, \ldots, m+1$ to which $y$ continues as it crosses the fold, and $\tau_{i}(m+1)$ is defined to be $y_{i}$.

The sequence of permutations $\left\{\sigma_{i}\right\}_{i=1}^{k},\left\{\tau_{i}\right\}_{i=1}^{k}$ characterizes the topology of the figure precisely. This is up to a symmetry factor which is related to the arbitrariness in the labeling of the $n+1$ sheets and the $m+1$ sheets, as well as the possibility to relabel the outer $n$ and $m$ sheets. To be more precise, it is (up to a factor) the number of permutations $\psi \in S_{n+1}$ and $\phi \in S_{n}$ such that $\sigma_{i}=\psi \sigma_{i} \phi$ for all $i$, times the number of permutations $\theta \in S_{m+1}$ and $\beta \in S_{m}$ such that $\tau_{i}=\theta \tau_{i} \beta$ for all $i$.

In general, when we pass from $\gamma_{i}$ to $\gamma_{i+1}$ not all the configurations $\left(\sigma_{i}, \tau_{i}\right) \rightarrow\left(\sigma_{i+1}, \tau_{i+1}\right)$ will be allowed. For example, we should require that

\footnotetext{
${ }^{2}$ We thank W. Taylor for correcting us with regards to this formula.
} 
$\left(x_{i+1}, y_{i+1}\right) \neq\left(x_{i}, y_{i}\right)$ as an ordered pair. If $\left(x_{i+1}, y_{i+1}\right)=\left(x_{i}, y_{i}\right)$ there is still the possibility to make a nontrivial permutation in the gluing of the inner sheets to the outer sheets, however this topological property cannot be assigned to a fold, i.e. a singularity along a line.

Furthermore, for general string theories (defined by the coupling constants) there will be different weights for different $\left(\sigma_{i}, \tau_{i}\right) \rightarrow\left(\sigma_{i+1}, \tau_{i+1}\right)$.

Let us denote by $F_{\sigma_{2} \tau_{2}}^{\sigma_{1} \tau_{1}}$ the weight that is attributed to the passage $\left(\sigma_{i}, \tau_{i}\right) \rightarrow\left(\sigma_{i+1}, \tau_{i+1}\right)$ along the fold. $F$ is a $(n+1) !(m+1) ! \times(n+1) !(m+1)$ ! symmetric matrix, which we shall call the fold-matrix.

The factor for a fold with $k$ singular points (and with the assumption that inside the area $A$ the boundaries of the $(n+1)+(m+1)$ sheets are disconnected is:

$$
\sum_{\sigma_{i}, \tau_{i}} S\left(\left\{\sigma_{i}\right\}_{i=1}^{k},\left\{\tau_{i}\right\}_{i=1}^{k}\right) F_{\sigma_{2} \tau_{2}}^{\sigma_{1} \tau_{1}} F_{\sigma_{3} \tau_{3}}^{\sigma_{2} \tau_{2}} \ldots F_{\sigma_{1} \tau_{1}}^{\sigma_{k} \tau_{k}}
$$

where the symmetry factors are 1 over the number of equivalence classes of $\left\{\sigma_{i}\right\}_{i=1}^{k},\left\{\tau_{i}\right\}_{i=1}^{k}$. It can be recast as

$$
\begin{aligned}
S\left(\left\{\sigma_{i}\right\}_{i=1}^{k},\left\{\tau_{i}\right\}_{i=1}^{k}\right) & =\frac{1}{(n+1) !(m+1) ! n ! m !} \\
& \cdot \sum_{\psi \in S_{n+1}, \phi \in S_{n}}\left(\prod_{i=1}^{k} \delta\left(\sigma_{i}^{-1} \psi \sigma_{i} \phi\right)\right) \\
& \cdot \sum_{\theta \in S_{m+1}, \beta \in S_{m}}\left(\prod_{i=1}^{k} \delta\left(\tau_{i}^{-1} \theta \tau_{i} \beta\right)\right)
\end{aligned}
$$

where $\delta$ is 1 if and only if its argument is the identity permutation. Thus the factor for $k$ singular points is:

$$
\frac{1}{(n+1) !(m+1) ! n ! m !} \sum_{\psi, \phi, \theta, \beta} \operatorname{Tr}\left(F_{(\psi, \phi, \theta, \beta)}(n, m)^{k}\right)
$$

where:

$$
\begin{aligned}
F_{(\psi, \phi, \theta, \beta)}(n, m) \stackrel{\text { def }}{=} & \left.\left.F_{\sigma_{2} \tau_{2}}^{\sigma_{1} \tau_{1}} \delta\left(\sigma_{1}^{-1} \psi \sigma_{1} \phi\right)\right) \delta\left(\sigma_{2}^{-1} \psi \sigma_{2} \phi\right)\right) \\
\cdot & \left.\left.\delta\left(\tau_{1}^{-1} \theta \tau_{1} \beta\right)\right) \delta\left(\tau_{2}^{-1} \theta \tau_{2} \beta\right)\right)
\end{aligned}
$$

We will call the $F_{(\psi, \phi, \theta, \beta)}(n, m)$-s the restricted fold matrices. 
The formula for the weight of a fold of length $l$ becomes:

$$
\begin{aligned}
& W=\sum_{k=0}^{\infty} \lambda_{s t}^{k-2(n+m+1)} \frac{l^{k}}{k !} \sum_{j} \sum_{\psi \in S_{n+1}, \phi \in S_{n}}\left(\alpha_{(\psi, \phi, \theta, \beta), j}(n, m)\right)^{k} \\
& \theta \in S_{m+1}, \beta \in S_{m} \\
& =\sum_{j} \sum_{\psi \in S_{n+1}, \phi \in S_{n}} \lambda_{s t}{ }^{-2(n+m+1)} e^{\lambda_{s t} \alpha_{(\psi, \phi, \theta, \beta), j}(n, m) l} \\
& \theta \in S_{m+1}, \beta \in S_{m}
\end{aligned}
$$

where $\alpha_{(\psi, \phi, \theta, \beta), j}(n, m)$ are the eigenvalues of the restricted fold-matrices. We have used (23) and absorbed the "spiralities" into the definition of the foldmatrix. The determination of the weight of the fold thus reduces to the determination of the eigenvalues of the restricted fold-matrices. In appendix A we will calculate the fold matrix for general $(n, m)$ under the assumption of no branch points on the folds (no spiralities). In appendix B we will show how to calculate the eigenvalues of the restricted fold matrices of appendix A.

We will end this subsection with a description of the slight modification that is needed when the boundaries of the $(n+1)+(m+1)$ sheets are not disconnected, as is the case when there are branch-points inside $A$ that do not cancel each other. When one goes along one sheet close to the fold boundary he will not return to the same sheet after one whole round, because there are branch cuts that start at the branch points inside the area bounded by the fold. Since the branch cuts can be defined arbitrarily, so long as they start at the branch points, we can make all the branch cuts pass through the fold segment between the last singular point and the first one. Note that when branch cuts are present singular points must exist when the fold is non-intersecting. Otherwise the fold will not close well. We will define permutations $\zeta \in S_{n+1}$ and $\xi \in S_{m+1}$ that describe how the $(n+1)$ (respectively $(m+1))$ sheets change to one another as one finishes a full round close to the boundary of $A$. In [3] the equivalence classes of $\zeta$ and $\xi$ were related to the representation in which a Wilson loop operator was taken around the boundary. 
Eqn.(24) is now modified to

$$
\sum_{\sigma_{i}, \tau_{i}} S\left(\left\{\sigma_{i}\right\}_{i=1}^{k},\left\{\tau_{i}\right\}_{i=1}^{k}\right) F_{\sigma_{2} \tau_{2}}^{\sigma_{1} \tau_{1}} F_{\sigma_{3} \tau_{3}}^{\sigma_{2} \tau_{2}} \ldots F_{\left(\zeta \sigma_{1}\right)\left(\xi \tau_{1}\right)}^{\sigma_{k} \tau_{k}}
$$

This can still be recast as a sum of eigenvalues if we write the product as $\operatorname{tr}\left(F^{k} P(\zeta, \xi)\right)$ where the matrix $P$ is given by

$$
P(\zeta, \xi)_{\sigma_{2} \tau_{2}}^{\sigma_{1} \tau_{1}}=\delta\left(\zeta \sigma_{1} \sigma_{2}^{-1}\right) \delta\left(\xi \tau_{1} \tau_{2}^{-1}\right)
$$

After diagonalizing the restricted fold matrices, the factor of the fold can still be written as a sum over exponents like (28) but with a factor in front of each exponent which is the diagonal element of $P$ in the basis in which the restricted fold matrix is diagonal.

We have to include also the effect of branch points in $\mathcal{M}-A$. (i.e. a nontrivial boundary for $\mathcal{M}-A$ near but a little bit to the outside of $\mathrm{A}$ ). This can be done similarly, by introducing permutations. We will define permutations $\zeta^{\prime} \in S_{n}$ and $\xi^{\prime} \in S_{m}$ that describe how the $n$ (respectively $m$ ) sheets change to one another as one finishes a full round close to the boundary of $\mathcal{M}-A$. We then have to insert

$$
P^{\prime}\left(\zeta^{\prime}, \xi^{\prime}\right)_{\sigma_{2} \tau_{2}}^{\sigma_{1} \tau_{1}}=\delta\left(\sigma_{1} \zeta^{\prime} \sigma_{2}^{-1}\right) \delta\left(\tau_{1} \xi^{\prime} \tau_{2}^{-1}\right)
$$

\subsection{Quartic Vertices}

The next step after considering the contribution to the partition function of a single fold is to consider the situation of several (possibly intersecting) folds. The figurative picture of a general map with folds is that of regions in the target space that are bounded by folds (see Fig.1 for example). We wish to develop "Feynman rules" to obtain the weight of such diagrams. Generic maps have folds, that are curves which intersect at certain points in which one fold passes above the other fold. Such points will be quartic vertices in the Feynman rules. Another source of quartic vertices is the quartic points of $(10)$.

In this subsection we obtain the contribution of the vertex and in the next subsection we will write down the full set of Feynman rules (in a formal form). 


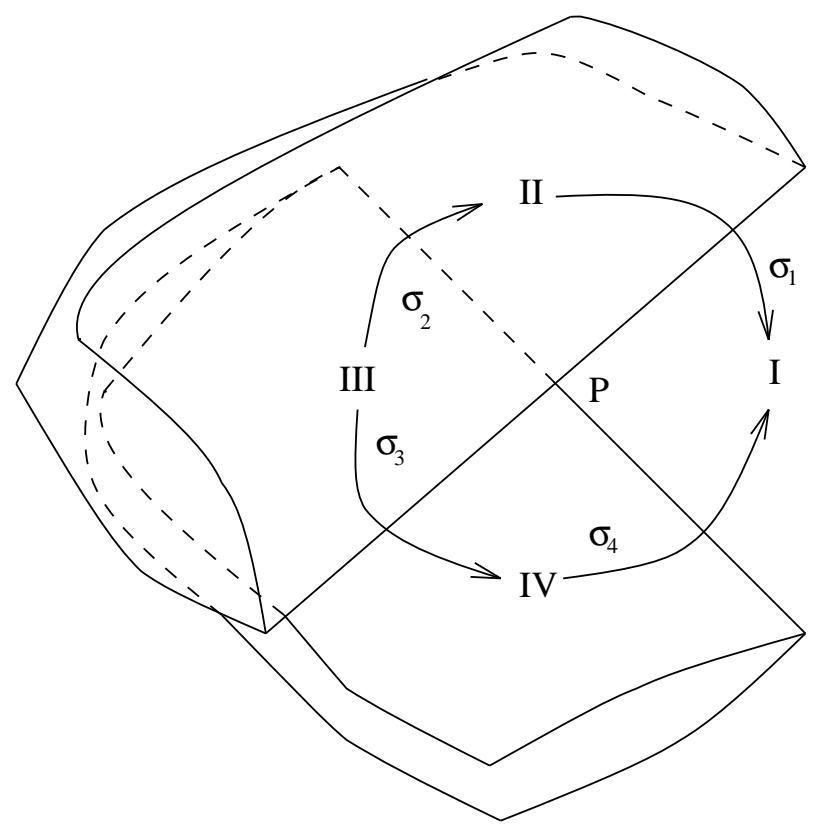

Fig. 12: A point where one fold passes on top of the other.

Let $P$ be a point at which one fold passes on top of the other (Fig.12). We will assume that the point $P$ itself is not a singular point on a fold, and thus we can restrict ourselves to a small neighborhood of $P$ in which there are no singular points on the folds. Near $P$ the folds divide the space into four regions with cover numbers $(n, m),(n+1, m+1),(n+1, m+1),(n+2, m+2)$. Let $\sigma_{1} \in S_{n+1}$ be the permutation that specifies how the $n$ sheets from region $I$ are continued to the sheets of region $I I$ (for some pre-determined labeling). We recall our convention that $\sigma_{1}(n+1)$ is the sheet in region $I I$ that is connected to the fold. similarly we define $\sigma_{4} \in S_{n+1}$ for the passage $I \rightarrow I V$ and $\sigma_{3}, \sigma_{2} \in S_{n+2}$ for the passages $I V \rightarrow I I I$ and $I I \rightarrow I I I$ respectively (see Fig.12). We define the permutations $\tau_{1}, \tau_{2}, \tau_{3}, \tau_{4}$ for the covers with the opposite orientation, in a similar fashion. Our requirement that $P$ be an ordinary point translates into the requirement that

$$
\sigma_{4}^{-1} \sigma_{3}^{-1} \sigma_{2} \sigma_{1}=P_{n+1, n+2}
$$


where $P_{n+1, n+2} \in S_{n+2}$ is the permutation that switches $(n+1)$ and $(n+2)$. The reason for this relation is as follows. After we make one loop around $P$, covers $1, \ldots, n$ in region $I$ return to themselves (otherwise $P$ would be a branch point). When we start with (the formal) cover number $(n+1)$ in region $I$ we surround the point $P$ in the following manner (see Fig.12): first we go over to the cover that is attached to the fold in region $I V$, we pass region $I I I$, we cross over to region $I I$ and then we meet the fold. So, by our conventions we end up with (the formal) cover number $(n+2)$. Similarly, for the $\tau$-s we have

$$
\tau_{4}^{-1} \tau_{3}^{-1} \tau_{2} \tau_{1}=P_{m+1, m+2}
$$

The quartic points of (10), on the other hand, satisfy either

$$
\begin{aligned}
\sigma_{4}^{-1} \sigma_{3}^{-1} \sigma_{2} \sigma_{1} & =P_{n+1, n+2} \\
\tau_{4}^{-1} \tau_{3}^{-1} \tau_{2} \tau_{1} & =(1)
\end{aligned}
$$

or

$$
\begin{aligned}
\sigma_{4}^{-1} \sigma_{3}^{-1} \sigma_{2} \sigma_{1} & =(1) \\
\tau_{4}^{-1} \tau_{3}^{-1} \tau_{2} \tau_{1} & =P_{m+1, m+2}
\end{aligned}
$$

where (1) is the identity permutation. These relations can be checked from (Fig.6) which corresponds to (10).

In the following section we describe a set of rules to calculate the weight that goes with a given division of the target-space into regions. The location of the folds is thus assumed to be fixed. In these rules we assume that no more than two folds intersect at any point of the target-space. In other words, if folds are considered as "propagators" of Feynman diagrams, then there are only quartic vertices and no higher ones. This is in agreement with the classical discussion in section (2.1), where it was claimed that a generic map has at most quartic singularities. We remark, however, that quantummechanically, "contact-terms" may arise and thus higher vertices, with more than two folds, may have to be considered.

\subsection{Feynman Rules}

The full weight of a configuration of folds (as in Fig.1 for example) is given by the following "Feynman Rules": 
1. cover numbers - associate with each region non-negative integer covernumbers $(n, m)$ in all possible ways, and such that when crossing each fold both $m$ and $n$ either increase by 1 or decrease by 1 .

2. vertices - near each vertex $P$ with folds $\gamma_{1}, \gamma_{2}, \gamma_{3}, \gamma_{4}$ such that $\gamma_{1}$ and $\gamma_{2}$ bound a region $A$ with cover-numbers $(n, m), \gamma_{2}$ and $\gamma_{3}$ bound a region $B$ with cover-numbers $(n+1, m+1), \gamma_{3}$ and $\gamma_{4}$ bound a region $C$ with cover-numbers $(n+2, m+2)$, and $\gamma_{4}$ and $\gamma_{1}$ bound a region $D$ with cover-numbers $(n+1, m+1)(B$ and $D$ are not necessarily different regions), introduce the following variables: $\sigma_{1} \in S_{n+1}$ and $\tau_{1} \in S_{m+1}$ on the fold $\gamma_{1}, \sigma_{2} \in S_{n+1}$ and $\tau_{2} \in S_{m+1}$ on the fold $\gamma_{2}, \sigma_{3} \in S_{n+2}$ and $\tau_{3} \in S_{m+2}$ on the fold $\gamma_{3}$, and $\sigma_{4} \in S_{n+2}$ and $\tau_{4} \in S_{m+2}$ on the fold $\gamma_{4}$. Adjoin a factor of

$$
\begin{aligned}
& \delta\left(\sigma_{4}^{-1} \sigma_{3}^{-1} \sigma_{2} \sigma_{1} P_{n+1, n+2}\right) \delta\left(\tau_{4}^{-1} \tau_{3}^{-1} \tau_{2} \tau_{1} P_{m+1, m+2}\right) \\
& \quad+\quad \lambda_{q p} \delta\left(\sigma_{4}^{-1} \sigma_{3}^{-1} \sigma_{2} \sigma_{1}\right) \delta\left(\tau_{4}^{-1} \tau_{3}^{-1} \tau_{2} \tau_{1} P_{m+1, m+2}\right) \\
& \quad+\quad \lambda_{q p} \delta\left(\sigma_{4}^{-1} \sigma_{3}^{-1} \sigma_{2} \sigma_{1} P_{n+1, n+2}\right) \delta\left(\tau_{4}^{-1} \tau_{3}^{-1} \tau_{2} \tau_{1}\right)
\end{aligned}
$$

where $\lambda_{q p}$ is the weight of quartic points as in (10) and is a free parameter of the model. The first term is the contribution of configurations where one fold just passes (without touching) on top of the other fold. We stress that renormalization may change the form of quartic vertices, and may add 6-vertices, 8-vertices and so on.

3. smooth regions - each smooth region gets a factor of $e^{-\mu A}$ where $A$ is the area of the region and $\mu$ is the cosmological constant. In addition, we can include branch points, collapsed handles and contracted tubes each with factors of $\lambda_{b r} A, \lambda_{c h} A$ and $\lambda_{c t} A$ where the $\lambda$-s are the corresponding coupling constants. Also every insertion contributes a corresponding power of $\lambda_{s t}$ - the string coupling constant, according to the change in world-sheet genus. An additional complexity that is caused by the branch points is the fact that the boundary of the region that contains branch points does no longer have to be made of $n$ (and $m$ ) distinct loops, but as we make a full loop around the region we may end up on a different sheet from the one we started with. Let $\xi \in S_{n}$ and $\zeta \in S_{m}$ denote the permutations that represent the changing of the sheets as we make a full round. These $\xi$-s and $\zeta$-s will have an effect on the symmetry-factor considerations and on the propagators. 
4. symmetry factors - adjoin with each smooth region of covers $(n, m)$ two permutations $\psi \in S_{n}$ and $\theta \in S_{m}$ that commute with the $\xi$ and $\zeta$ that where associated with that region if it has branch points (otherwise the $\xi$ and $\zeta$ can be taken as the identity).

5. propagators - give each fold a factor of

$$
\left(\exp \left(\lambda_{s t} l F_{(\psi, \phi, \theta, \beta)}(n, m)\right)\right)_{\sigma_{2} \tau_{2}}^{\sigma_{1} \tau_{1}}
$$

where $F$ is the restricted fold matrix (see (27)), $\psi$ and $\theta$ are the symmetryfactor permutations associated with the region on one side of the fold, and $\phi$ and $\beta$ are those associated with the other side of the fold. $(n, m)$ are the cover numbers of the region with the fewer covers. $\sigma_{1}$ and $\tau_{1}$ are the permutations that were written on the fold near the first vertex that bounds it and $\sigma_{2}$ and $\tau_{2}$ were written on the fold near the second vertex that bounds it. $l$ is the length of the fold and $\lambda_{s t}$ is the string coupling constant.

6. branch cuts - There is a further complication because of the $\xi$-s and $\zeta$-s of the smooth region. For each smooth region we must pick one propagator (i.e. fold) that bounds it, and insert a change of the a labeling of the covers of the region at a certain point. This is done by replacing $\left(\exp \left(\lambda l F_{(\psi, \phi, \theta, \beta)}(n, m)\right)\right)_{\sigma_{2} \tau_{2}}^{\sigma_{1} \tau_{1}}$ for that propagator, with

$$
\left(\exp \left(\lambda l_{2} F_{(\psi, \phi, \theta, \beta)}(n, m)\right) P(\xi, \zeta) \exp \left(\lambda l_{1} F_{(\psi, \phi, \theta, \beta)}(n, m)\right)\right)_{\sigma_{2} \tau_{2}}^{\sigma_{1} \tau_{1}}
$$

where $l_{1}$ and $l_{2}$ are the lengths from the vertices to the point where the cut intersects the fold. The matrix $P$ is given by

$$
P(\zeta, \xi)_{\sigma_{2} \tau_{2}}^{\sigma_{1} \tau_{1}}=\delta\left(\zeta \sigma_{1} \sigma_{2}^{-1}\right) \delta\left(\xi \tau_{1} \tau_{2}^{-1}\right)
$$

when $P$ corresponds to a permutation due to a cut from branch points from the side of the fold where there are more sheets, and is given by

$$
P\left(\zeta^{\prime}, \xi^{\prime}\right)_{\sigma_{2} \tau_{2}}^{\sigma_{1} \tau_{1}}=\delta\left(\sigma_{1} \zeta^{\prime} \sigma_{2}^{-1}\right) \delta\left(\tau_{1} \xi^{\prime} \tau_{2}^{-1}\right)
$$

when $P$ corresponds to a permutation due to a cut from branch points From the side of the fold where there are less sheets.

7. statistics of closed loops - our remark after formula (23) about the contribution of the covers to the genus means that we have to add a factor of $\lambda_{s t}{ }^{2}$ for each closed loop (of folds). 
8. taking the trace - sum over all permutations $\sigma_{i}, \tau_{i}$ and the permutations $\psi$-s and $\theta$-s.

$$
\begin{aligned}
& (\psi, \theta) \\
& (n+1, m+1) \\
& \left(e^{\lambda l F_{(\psi, \phi, \theta, \beta)}(n, m)}\right)_{\sigma_{2}, \tau_{2}}^{\sigma_{1}, \tau_{1}} \\
& \sigma_{1}, \tau_{1} \frac{(n+1, m+1)}{(n, m)} \sigma_{2}, \tau_{2} \\
& \delta\left(\sigma_{4}^{-1} \sigma_{3}^{-1} \sigma_{2} \sigma_{1} P_{n+1, n+2}\right) \delta\left(\tau_{4}^{-1} \tau_{3}^{-1} \tau_{2} \tau_{1} P_{m+1, m+2}\right) \\
& +\lambda_{q p} \delta\left(\sigma_{4}^{-1} \sigma_{3}^{-1} \sigma_{2} \sigma_{1}\right) \delta\left(\tau_{4}^{-1} \tau_{3}^{-1} \tau_{2} \tau_{1} P_{m+1, m+2}\right) \\
& +\lambda_{q p} \delta\left(\sigma_{4}^{-1} \sigma_{3}^{-1} \sigma_{2} \sigma_{1} P_{n+1, n+2}\right) \delta\left(\tau_{4}^{-1} \tau_{3}^{-1} \tau_{2} \tau_{1}\right) \\
& \delta\left(\zeta \sigma_{1} \sigma_{2}^{-1}\right) \delta\left(\xi \tau_{1} \tau_{2}^{-1}\right) \\
& \sigma_{3}, \tau_{3}(n+2, m+2)>^{\sigma_{4}, \tau_{4}} \\
& \delta\left(\sigma_{1} \zeta^{\prime} \sigma_{2}^{-1}\right) \delta\left(\tau_{1} \xi^{\prime} \tau_{2}^{-1}\right) \\
& \sigma_{1}, \tau_{1} \frac{(n+1, m+1)}{\text { cut }} \sigma_{2}, \tau_{2}
\end{aligned}
$$

Fig. 13: Feynman rules. 


\section{Summing over fold configurations and sim- ple cases on a lattice}

So far we have concentrated on the contribution to the partition function from a fixed fold configuration (locus of singular points) in the target space. We saw that the contribution of all maps with the specified fold configuration reduces to a combinatorial calculation for which we had the Feynman rules of the previous section. In order to obtain the full partition function we have to sum over all the fold configurations as well. We do not know how to perform this task in general. We also have to admit that in the Feynman rules of the previous chapter there is no small parameter that limits the number of fold intersections.

In this section, however, we will demonstrate what is involved in the (maybe) simpler problem of summing over fold configurations which separate $(1,1)$ regions from $(0,0)$ regions in a toroidal target space. We assume that no higher $(m, n)$ regions exist and thus the "combinatorial" contribution is trivial. In this case the geometrical picture is that of Riemann surfaces of various genera smashed on a spherical target space in a non-overlapping way. The problem is to sum over all such configurations with the weight of

$$
\lambda_{s t} \sum_{i}\left(2-2 g_{i}\right) e^{-2 \mu \sum_{i} A_{i}}
$$

where the $A_{i}$-s are the areas of the bounded $(1,1)$ regions and the $g_{i}$-s are the genera of the smashed surfaces which correspond to the bounded regions. We will formulate the problem on a lattice and map the lattice problem to a perturbed Ising model with a triple interaction round a face. This model is known as the Baxter-Wu model[12].

It turns out to be convenient to work on a hexagonal lattice. We will thus pick a hexagonal lattice $\Gamma$ for the target space, with hexagons as faces. We put spin variables $\sigma_{k}= \pm 1$ on each face and interpret a given configuration of $\left\{\sigma_{k}\right\}$ as a partition of the target space into regions in such a way that the hexagons $k$ with $\sigma_{k}=+1$ belong to the $(1,1)$ regions and the hexagons with $\sigma_{k}=-1$ belong to the $(0,0)$ regions. The index $k$ is a vertex of the dual lattice $\Gamma^{*}$. The total area is given by

$$
2 \sum_{i} A_{i}=\sum_{k \in \Gamma^{*}}\left(1+\sigma_{k}\right)
$$

\footnotetext{
${ }^{3}$ We thank A. Zamolodchikov for pointing our attention to this reference
} 
The Euler characteristic of the figure $\sum_{i}\left(2-2 g_{i}\right)$ is given by Euler's formula $V+F-E$ where we use the triangulation that is given by the lattice $\Gamma$. For the number of faces $F$ we count the number of hexagons in a $(1,1)$ region with weight 2 . Note that hexagons in a $(0,0)$ region do not contribute to $F$. For the number of edges $E$ we count an edge twice if it is inside a $(1,1)$ region, we count it once if it is on the boundary between a $(1,1)$ and a $(0,0)$ region and we don't count it if it is in a $(0,0)$ region. We will use the relation $E=3 F$ (since every face has six different edges and every edge is common to two faces. For the vertices $V$ we again count a vertex twice inside a $(1,1)$ region, once on the boundary and don't count a vertex inside a $(0,0)$ region. We can express these relations in terms of the spin variables $\left\{\sigma_{k}\right\}$ as follows:

$$
\begin{aligned}
\sum_{i}\left(2-2 g_{i}\right) & =V+F-E=V-2 F \\
F & =N_{\text {faces }}+\sum_{k \in \Gamma^{*}} \sigma_{k} \\
V & =\sum_{\Delta_{k l m} \in \Gamma^{*}}\left(1-\frac{1}{4}\left(\sigma_{k}+\sigma_{l}+\sigma_{m}+\sigma_{k} \sigma_{l} \sigma_{m}\right)\right) \\
& =N_{\text {vertices }}-\frac{3}{2} \sum_{k \in \Gamma^{*}} \sigma_{k}-\frac{1}{4} \sum_{\Delta_{k l m} \in \Gamma^{*}} \sigma_{k} \sigma_{l} \sigma_{m}
\end{aligned}
$$

where $N_{\text {faces }}$ is the number of faces in the lattice $\Gamma$ and $N_{\text {vertices }}$ is the number of vertices in $\Gamma . \Delta_{k l m} \in \Gamma^{*}$ is an elementary triangular cell in $\Gamma^{*}$ with vertices $k, l, m$.

Thus we obtain

$$
\begin{aligned}
e^{-2 \mu \sum_{i} A_{i}} \lambda_{s t} \sum_{i}\left(2-2 g_{i}\right) & =e^{-\left(\mu+2 \log \lambda_{s t}\right) N_{\text {faces }}+\log \lambda_{s t} N_{\text {vertices }}} \\
\cdot \exp \left\{-\left(\mu+\frac{7}{2} \log \lambda_{s t}\right) \sum_{k \in \Gamma^{*}} \sigma_{k}\right. & \left.-\frac{1}{4} \log \lambda_{s t} \sum_{\Delta_{k l m} \in \Gamma^{*}} \sigma_{k} \sigma_{l} \sigma_{m}\right\}
\end{aligned}
$$

Thus the Hamiltonian is given by

$$
\mathcal{H}=-\left(\mu+\frac{7}{2} \log \lambda_{s t}\right) \sum_{k \in \Gamma^{*}} \sigma_{k}-\frac{1}{4} \log \lambda_{s t} \sum_{\Delta_{k l m} \in \Gamma^{*}} \sigma_{k} \sigma_{l} \sigma_{m}
$$

This is the Hamiltonian of the Baxter-Wu model in an external magnetic field [12].

We will recall the results of [13] and briefly describe their consequences here: 
1. The model with hamiltonian $K \sum_{\Delta_{k l m} \in \Gamma^{*}} \sigma_{k} \sigma_{l} \sigma_{m}$ has a second order phase transition for $K_{c}= \pm 0.4406 \ldots$ and zero magnetic field. In our case, we get

$$
\lambda_{s t}=0.171 \ldots, \quad \mu=6.168 \ldots
$$

(Recall that $\mu$ is the cosmological constant per lattice cell.)

2. Near the critical point let $\Delta K=K-K_{c}$. Then, in the vicinity of $K_{c}$, the specific heat of the model behaves as $|\Delta K|^{-\frac{2}{3}}$. The spontaneous magnetization for $K<K_{c}$ behaves as $|\Delta K|^{\frac{1}{12}}$. It can be shown from scaling assumptions [13] that the partition function behaves as $\log Z \sim$ $|\Delta K|^{\frac{4}{3}} F\left(H|\Delta K|^{-\frac{5}{4}}\right)$ where $H$ is a small magnetic field and $F$ is some function (which is different for $\Delta K>0$ and $\Delta K<0$ ). In our case, we replace $|\Delta K|$ by $\Delta \lambda_{s t}$ and $H$ by $\Delta \mu$ (since $H$ scales as $\left|\Delta \lambda_{s t}\right|^{\frac{5}{4}}$ the extra $\frac{7}{2} \log \lambda_{s t}$ in (3) do not contribute to the scaling behavior). We get

$$
\log Z \sim\left|\Delta \lambda_{s t}\right|^{\frac{4}{3}} F\left(\Delta \mu\left|\Delta \lambda_{s t}\right|^{-\frac{5}{4}}\right)
$$

3. At the critical point itself the correlation functions behave as $r^{-\frac{1}{4}}$. Returning to the string model, we can deduce the tachyonic two-point functions. Denoting by $Y$ a target space coordinate we can write the operator that corresponds to the tachyon as the Fourier transform of the cover number at $Y$ namely, $T_{k}=\int d^{2} Y e^{k \cdot Y} n(Y)$ where $n(Y)=$ $\int d^{2} \xi \delta(Y-X(\xi)) d^{2} \xi$. A two-point function of tachyons can be rewritten as $\left\langle T_{k} T_{-k}\right\rangle=\int d^{2} Y e^{k \cdot Y}\langle n(Y) n(0)\rangle$. The restriction to unfolded maps makes $n(Y)$ independent of $Y$ and therefore, all the two point functions (apart from $T_{0}$ ) vanish. In the presence of folds the cover numbers at different target space points are correlated and the tachyon correlators are non-trivial. In our case we get

$$
\langle n(Y) n(0)\rangle=1+2\langle\sigma\rangle+\langle\sigma(Y) \sigma(0)\rangle
$$

and using $\langle n(Y) n(0)\rangle \sim|Y|^{-\frac{1}{4}}$ we get the tachyon two-point function for $k \neq 0$

$$
\left\langle T_{k} T_{-k}\right\rangle \sim|k|^{-\frac{7}{4}}
$$

At this stage we do not know how to interpret this result in comparison with the corresponding correlators of string models. 


\section{Interpretation of folds as particle trajecto- ries}

The string theory that describes the large $N$ limit of $S U(N)$ 2D Yang-Mills theory was developed in [3] (and following it in [0] for $U(N)$ ). it is a theory of maps with no folds. The string coupling constant is identified with $1 / N$, the string tension is given by $\lambda / 2$ where $\lambda$ is related to the gauge coupling $\tilde{g}$ by $\lambda=\tilde{g}^{2} N$. Maps with a branch point in an infinitesimal area $d A$ have a weight of $\lambda d A$. There is, however, a mysterious feature of this string theory which is the $2-2 G-B$, where $G$ is the target space genus and $B$ is the number of its boundary components, constant points in the target space. f $^{-}$

For these points the maps are allowed to have an arbitrary branch point connecting arbitrary sheets and have weight 1 instead of an infinitesimal weight of $\lambda d A$.

We wish to incorporate folds and singular points in this framework. Let's consider a sphere target space with a simple fold that divides the target space into two simply connected areas $A$ and $\mathcal{M}-A$. According to [3] the natural operators which capture the geometry at the boundary $\partial A$ are the "generalized Frobenius characters" which are given by equation (A.7) of [3]

$$
\begin{gathered}
\Upsilon_{\bar{\tau} \sigma}\left(U, U^{\dagger}\right)=\sum_{v \subset T_{\sigma}} \sum_{v^{\prime} \subset T_{\tau}, v \approx v^{\prime}}(-1)^{K_{v}} C_{v} \Upsilon_{\sigma \backslash v}(U) \Upsilon_{\tau \backslash v^{\prime}}\left(U^{\dagger}\right) \\
=\sum_{v_{1}, v_{2}, \ldots}(-1)^{\sum v_{i}} \prod_{i}\left(i^{v_{i}} v_{i} !\left(\begin{array}{c}
\sigma_{i} \\
v_{i}
\end{array}\right)\left(\begin{array}{c}
\tau_{i} \\
v_{i}
\end{array}\right)\right) \prod_{i}\left(\operatorname{Tr} U^{\sigma_{i}-v_{i}}\right) \prod_{i}\left(\operatorname{Tr}\left(U^{\dagger}\right)^{\tau_{i}-v_{i}}\right)
\end{gathered}
$$

where $\sigma$ and $\tau$ are permutations, $T_{\sigma}$ and $T_{\tau}$ are the conjugacy classes of $\sigma$ and $\tau$ respectively and $K_{\sigma}$ is the number of cycles of $\sigma . C_{v}$ is given by

$$
C_{v}=\prod_{i} i^{v_{i}} v_{i} !
$$

where $v_{i}$ is the number of cycles in $v$ of length $i$, and

$$
\Upsilon_{\sigma}(U)=\prod_{i=1}^{K_{\sigma}}\left(\operatorname{Tr} U^{k_{i}}\right)
$$

\footnotetext{
${ }^{4}$ Those points have lately received a new interpretation in [10].
} 
where $k_{i}$ is the length of the $i$-th cycle of $\sigma$.

According to equation (4.8) of [3] the functional integral of the YangMills action on one side of the fold (say A) times the value of $\Upsilon_{\bar{\tau} \sigma}\left(U, U^{\dagger}\right)$, where $U$ is the holonomy of the gauge field around $\partial A$, is given by the sum of maps that cover $A$ so that the boundary is made up of $K_{\sigma}+K_{\tau}$ components. $K_{\sigma}$ components that are boundaries of sheets of positive orientation and $K_{\tau}$ components that are boundaries of sheets of the opposite orientation. The $i$-th component from the $K_{\sigma}$ has a winding of $\sigma_{i}$ and the $j$-th component from the $K_{\tau}$ has a a winding of $\tau_{j}$. When there is no fold on $\partial A A$ and $\mathcal{M}-A$ are glued by the gluing formula (4.14) of [3]

$$
\int d U \Upsilon_{\bar{\tau} \sigma}\left(U, U^{\dagger}\right) \Upsilon_{\bar{\tau}^{\prime} \sigma^{\prime}}\left(U^{\dagger}, U\right)=\delta_{T_{\sigma}, T_{\sigma^{\prime}}} \delta_{T_{\tau^{\prime}}, T_{\tau}} C_{\sigma} C_{\tau}
$$

This means that the boundary of $A$ (which is parameterized by $T_{\sigma}$ and $T_{\tau}$ ) is glued to the boundary of $\mathcal{M}-A$ (which is parameterized by $T_{\sigma^{\prime}}$ and $T_{\tau^{\prime}}$ ) so that each $k$-cycle of $\sigma$ is attached to a $k$-cycle of $\sigma^{\prime}$ and each $k$-cycle of $\tau$ is attached to a $k$-cycle of $\tau^{\prime}$.

We wish to find the matrix elements of a fold between the states $\left\langle\sigma^{\prime} \tau^{\prime}\right|$ and $|\sigma \tau\rangle$. The relevant target space is a cylinder whose upper boundary corresponds to the permutation classes $T_{\sigma^{\prime}}$ and $T_{\tau^{\prime}}$ and whose lower boundary corresponds to $T_{\sigma}$ and $T_{\tau}$. In the middle of the cylinder there is a fold (see Fig.14).

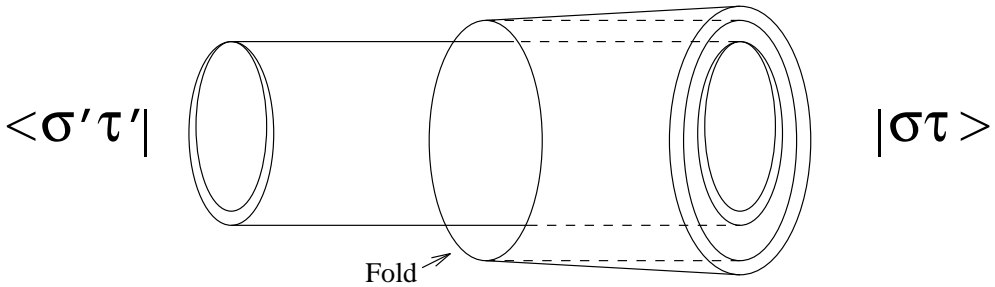

Fig. 14: The matrix element of a fold on a cylindrical target space.

If $\sigma \in S_{n}$, let $D_{k} \sigma \in S_{n+k}$ denote a permutation in the conjugacy class that is obtained from the conjugacy class of $\sigma$ by adding one extra $k$-cycle. Similarly let $D_{k}^{-1} \sigma \in S_{n-k}$ denote a permutation in the conjugacy class that 
is obtained from the conjugacy class of $\sigma$ by extracting one $k$-cycle. A fold with no singular points can either add one 1-cycle to $\sigma$ and one 1-cycle to $\tau$ or take away one 1-cycle from $\sigma$ and one 1-cycle from $\tau$. Let $\mathcal{O}_{F}$ denote the operator of the fold. Then

$$
\mathcal{O}_{F}|\sigma, \tau\rangle=\left|D_{1} \sigma, D_{1} \tau\right\rangle+\sigma_{1} \tau_{1}\left|D_{1}^{-1} \sigma, D_{1}^{-1} \tau\right\rangle
$$

where the $\sigma_{1} \tau_{1}$ coefficient in the second term is because we can take out any one of the $\sigma_{1}$ 1-cycles of $\sigma$ and any one of the $\tau_{1} 1$-cycles of $\tau$.

From (39) we see that

$$
\begin{aligned}
& \operatorname{Tr}(U) \operatorname{Tr}\left(U^{\dagger}\right) \Upsilon_{\bar{\tau} \sigma}\left(U, U^{\dagger}\right)=\Upsilon_{\overline{D_{1} \tau} D_{1} \sigma}\left(U, U^{\dagger}\right)+\sigma_{1} \tau_{1} \Upsilon_{\overline{D_{1}^{-1} \tau} D_{1}^{-1} \sigma}\left(U, U^{\dagger}\right) \\
& +\left(\sigma_{1}+\tau_{1}+1\right) \Upsilon_{\bar{\tau} \sigma}\left(U, U^{\dagger}\right)
\end{aligned}
$$

where we have used the combinatorial identity

$$
\begin{aligned}
& \sum_{v}(-1)^{v}\left(\begin{array}{l}
a \\
v
\end{array}\right)\left(\begin{array}{l}
b \\
v
\end{array}\right) v ! x^{a+1-v} y^{b+1-v}= \\
& (a+b+1) \sum_{v}(-1)^{v}\left(\begin{array}{l}
a \\
v
\end{array}\right)\left(\begin{array}{l}
b \\
v
\end{array}\right) v ! x^{a-v} y^{b-v} \\
& +a b \sum_{v}(-1)^{v}\left(\begin{array}{c}
a-1 \\
v
\end{array}\right)\left(\begin{array}{c}
b-1 \\
v
\end{array}\right) v ! x^{a-1-v} y^{b-1-v} \\
& +\sum_{v}(-1)^{v}\left(\begin{array}{c}
a+1 \\
v
\end{array}\right)\left(\begin{array}{c}
b+1 \\
v
\end{array}\right) v ! x^{a+1-v} y^{b+1-v}
\end{aligned}
$$

for $x=\operatorname{Tr}(U), y=\operatorname{Tr}\left(U^{\dagger}\right), a=\sigma_{1}$ and $b=\tau_{1}$. Equation (40) expresses the fact that the two boundaries of opposite orientations that are created by the operator $\operatorname{Tr}(U) \operatorname{Tr}\left(U^{\dagger}\right)$, can be formed by either a fold in the upward direction (the first term in the rhs), a fold that goes downward (the second term), or without a fold - by cutting a sheet into two (the last term). We get the symbolic relation

$$
\mathcal{O}_{F}=\left(\operatorname{Tr}(U) \operatorname{Tr}\left(U^{\dagger}\right)-1\right)-\left(\sigma_{1}+\tau_{1}\right)
$$

If it were only the $\operatorname{Tr}(U) \operatorname{Tr}\left(U^{\dagger}\right)-1$ term, we could understand a fold as a trajectory of a particle in the adjoint representation of $S U(N)$ (or $U(N)$ ) since it would carry the weight

$$
\operatorname{Tr}(U) \operatorname{Tr}\left(U^{\dagger}\right)-1=\operatorname{Tr}_{A d j}\left(P e^{i \oint A^{\alpha} d x_{\alpha}}\right)
$$


The second term $\sigma_{1}+\tau_{1}$ means that we have to add a particle which is a singlet ghost (because of the (-) sign) and has a peculiar interaction with the gauge field such that it has a multiplicity of $\sigma_{1}+\tau_{1}$. At this stage the nature of this apparent ghost is not clear to us.

Next, we consider a fold on the cylinder with one singular point on it. Suppose that there are two more sheets above the fold. Denote by $(n, m)$ the numbers of sheets below the fold, whose boundary components are parameterized by $\sigma$ and $\tau$. We can pick some labeling of the $(n+m)$ sheets at the singular point $P$ on the fold, and then $\sigma$ and $\tau$ will not be just conjugacy classes but will be unique permutations that specify how the sheets change after a permutation starting at $P$. We will work with the following settings: To the left of $P$ sheet ' $a$ ' is connected to the fold and sheet ' $b$ ' goes over to sheet ' $x$ ' below the fold. To the right of $P$ sheet ' $b$ ' is connected to the fold and sheet ' $a$ ' goes over to sheet ' $x$ '. If ' $x$ ' is one of the $n$ orientability $(+)$ sheets, then the fold changes $|\bar{\tau} \sigma\rangle$ into $\left|\bar{\tau} \rho_{x, n+1} \sigma\right\rangle$ where $\rho_{x, n+1} \in S_{n+1}$ is the permutation that switches the two elements $x$ and $n+1$. Similarly, when $x$ is one of the $m$ orientability $(-)$ sheets, the fold changes $|\bar{\tau} \sigma\rangle$ into $\left|\overline{\rho_{x, m+1} \tau} \sigma\right\rangle$. So we can write symbolically the operator that corresponds to the singular point $P$ on a fold that is facing downwards as:

$$
\mathcal{O}_{P}=\sum_{x} \rho_{x, n+1}+\sum_{y} \rho_{y, m+1}
$$

but

$$
\rho_{x, n+1}=\sum_{x<y \leq n+1} \rho_{x, y}-\sum_{x<y \leq n} \rho_{x, y}
$$

The operator $\sum_{x<y \leq n} \rho_{x, y}$ is related to the operator $\operatorname{Tr}\left(F\left(P_{-}\right)^{2}\right)$ where $F(P)$ is the YM field strength at the point $P$ and the subscript $P_{-}$indicates that we actually have to take the field strength a little bit below the point $P$ (that is, a little bit below the fold). To see the relation we note that if there was no fold, the string operator $\sum_{x<y \leq n} \rho_{x, y}$ changes a boundary state of $|\bar{\tau} \sigma\rangle$ as follows

$$
|\bar{\tau} \sigma\rangle \rightarrow \sum_{x<y \leq n}\left|\overline{\rho_{x, y} \tau} \sigma\right\rangle
$$

Thus its matrix elements are given precisely by a sum of maps that have a branch point at $P$ (and the weights of the maps are according to the rules of [3]). By differentiating the equation for the partition function of $Y M_{2}, \mathrm{u}$ 
written in terms of maps (equation (3.6) of [3]) with respect to $\lambda$ we obtain the string analog of the operator insertion of $\operatorname{Tr}\left(F^{2}\right)$ at a point $P$ :

$$
\frac{N}{4 \lambda^{2}} \operatorname{Tr}\left(F^{2}\right)=-\frac{1}{2}(n+m)+\frac{(n-m)^{2}}{2 N^{2}}-\frac{1}{N}\left(\sum_{x<y \leq n} \rho_{x, y}+\sum_{\bar{x}<\bar{y} \leq m} \rho_{\bar{x}, \bar{y}}\right)
$$

The last term has the following meaning: when it is inserted in expectation values (of Wilson loops) it counts the maps with a branch point at $P$. It arises from the contribution of $\lambda$ to the weight of maps with branch points at $P . n(P)$ and $m(P)$ are the number of covers of $(+)$ and $(-)$ orientability at $P$. To get $(\boxplus)$ we summed over connecting tubes and contracted handles at $P$. Thus we see that

$$
\sum_{x<y \leq n} \rho_{x, y}+\sum_{\bar{x}<\bar{y} \leq m} \rho_{\bar{x}, \bar{y}}=-\frac{N^{2}}{4 \lambda^{2}} \operatorname{Tr}\left(F^{2}\right)+N \frac{1}{2}(n+m)-\frac{(n-m)^{2}}{2 N}
$$

We thus get the following expression for the operator of a singular point on a fold

$$
\mathcal{O}_{P}=2 N-\frac{N^{2}}{4 \lambda^{2}}\left(\operatorname{Tr}\left(F\left(P_{+}\right)^{2}\right)-\operatorname{Tr}\left(F\left(P_{-}\right)^{2}\right)\right.
$$

where $P_{+}$means a little bit above the fold and $P_{-}$means a little bit below the fold.

Suppose the operator $\mathcal{O}_{F}$ of a fold with no singular points was just $\operatorname{Tr}(U) \operatorname{Tr}\left(U^{\dagger}\right)-1$, which is the trace in the adjoint representation of the Wilson loop along the fold. This would mean, as mentioned above, that the interaction of a fold with the gauge field is exactly like that of a particle in the adjoint representation of an $S U(N)$ gauge group. In that case the operator $\mathcal{O}_{P}$ would give a mass insertion of $2 N$ and a peculiar interaction with the jump in $\operatorname{Tr}\left(F^{2}\right)$ along the fold.

Another QCD operator that may be related to the operator $\mathcal{O}_{P}$ is an insertion of $g_{\mu \nu} D_{\tau} F^{\tau \nu}$. This operator is explicitly not APD invariant and is inserted into Wilson loops as follows

$$
\operatorname{Tr}_{A d j} P e^{\oint A_{\mu} d x^{\mu}} \rightarrow \operatorname{Tr}_{A d j} P e^{\oint\left(A_{\mu}+\rho g_{\mu \nu} D_{\tau} F^{\tau \nu}\right) d x^{\mu}}
$$

We can interpret this insertion pictorially. Consider a Wilson loop in the adjoint. As mentioned above, according to [3] there are three groups of string maps that are to be considered. Those that have two more sheets to 
one side of the Wilson loop, those that have two more sheets to the other side, and those that have the same number of sheets on both sides.

An insertion of $g_{\mu \nu} D_{\tau} F^{\tau \nu}$ at a point $x$ can be interpreted as deforming one of the two boundaries of the Wilson loop at the point $x$ slightly outwards (say by a square) and then subtracting a similar deformation slightly inwards, and taking the $O(\Delta A)$ coefficient [14]. In the string picture the maps that contribute to the $O(\Delta A)$ are those that have a branch point in the $\Delta A$ region, and there is also a contribution from the $e^{\lambda \Delta A}$ term for maps which do not necessarily have a branch point in the $\Delta A$ region. The problem is that we get a contribution with a negative sign for the maps that have two more sheets on the other side!

We remark, that the $\Omega$-points of [3] do not cause a real difficulty since every boundary component creates one $\Omega$-point on one side of it and an $\Omega^{-1}$ point on the other side. Thus, a fold that has one boundary from every orientation creates a pair of an $\Omega$-point and an $\Omega^{-1}$-point on each side of it, and the pairs cancel.

We will end by summarizing the discrepancies that are still left in the interpretation of folds as particle trajectories.

- The correspondence between maps with a fold boundary and maps that calculate the expectation value of a Wilson loop in the adjoint representation is not complete because the latter include maps that are described by cutting a smooth sheet along a trajectory (thus creating a two boundaries of opposite orientations) and not just by maps that are obtained by cutting along a fold. The extra maps are described by the $\sigma_{1}+\tau_{1}$ term of (田).

- The peculiar interaction of the fold with the discontinuity of $\operatorname{Tr}\left(F^{2}\right)$ along it does not have a satisfactory Lagrangian description.

- The quartic vertices of the "Feynman diagrams" of the previous section were not explained in terms of a lagrangian.

\section{$7 \quad$ Summary and Discussion}

In this paper we have explored the role of folded maps in two dimensional string theory. We have removed several obstacles on the way toward computing the contribution of folds to the string partition function. In this respect 
we have made a certain progress in the program of quantizing the $2 \mathrm{D} \mathrm{NG}$ theory. Folds have been shown to admit a behavior of particles with special interaction with non-abelian gauge fields. This may turn out to be relevant in the search of stringy nature of $Q C D_{2}$.

The precise relation between the NG theory and the Polyakov string theory at the quantum level has not yet been fully clarified. In $2 \mathrm{D}$ one expects that the non-critical string theory of $c=1$ is related to the NG theory [1]. This correspondence faces several problems, for instance what Liouville theory corresponds to higher genus 2D target space.

To the extent that the correspondence contains certain truth, one would associate the NG folds with the massless tachyons of the Liouville theory. Indeed our analysis partially supports such an interpretation. Denoting by $Y$ a target space c-number, we can write the operator that corresponds to the tachyon as the Fourier transform of the cover number at $Y$ namely, $T_{k}=\int d^{2} Y e^{k \cdot Y} n(Y)$ where $n(Y)=\int d^{2} \xi \delta(Y-X(\xi)) d^{2} \xi$. A two-point function of tachyons can be rewritten as $\left\langle T_{k} T_{-k}\right\rangle=\int d^{2} Y e^{k \cdot Y}\langle n(Y) n(0)\rangle$. The restriction to unfolded maps makes $n(Y)$ independent of $Y$ and therefore, all the two point functions (apart from $T_{0}$ ) vanish. In the presence of folds the cover numbers at different target space points are correlated and the tachyon correlators are non-trivial. It is easy to realize that tachyons indeed have vanishing $\operatorname{det} J$. It seems that they are associated with a fold- "antifold" configuration.

In the present work we have put forward the idea that folds should be associated with particle trajectories. At present we have certain evidence that support it, even though, a full understanding of the nature of those particles is still ahead of us. The contribution to the partition function of a single fold enclosing a region covered with three sheets resembles the contribution of a particle with mass $\lambda_{s t} \rho$. Moreover, an analysis based on the methods of [3] indicates that indeed a fold could be understood as a trajectory of a particle in the adjoint representation with certain peculiar interaction with the gauge fields. So far this peculiar interaction of the fold with the discontinuity of $\operatorname{Tr}\left(F^{2}\right)$ along it does not have a satisfactory Lagrangian description. A full correspondence between maps with a fold boundary and maps that calculate the expectation value of a Wilson loop in the adjoint representation was not established. For the case of a Wilson loop the maps are described by cutting a smooth sheet along a trajectory (thus creating a two boundaries of opposite orientations) and not just by maps that are obtained by cutting 
along a fold. The extra maps are described by the $\sigma_{1}+\tau_{1}$ term of (44). Clearly, this important issue deserves more study.

The stringy picture of $Y M_{2}$ is based on the perturbative $\frac{1}{N}$ expansion. The weighted sum over unfolded maps was constructed to reproduce this expansion. So far we lack a stringy action which encodes this sum over maps. Recently, some progress has been made toward this goal. First, it was argued [5] that to account for the $U(N)$ (rather than $S U(N)$ ) partition function, the only point singularities which appear in the maps are branch points and the so called " $\Omega$-points". One has to insert $2 G-2$ of the latter and, therefore, for a target space with topology of a torus $(G=1)$, only branch points are left. In this case, summing over holomorphic maps from a torus world sheet reproduces the chiral $Y M_{2}$ partition function[8]. The sum over holomorphic maps for a toroidal target space is encoded in the topological $A$ type sigma model[15]. A proposal following these lines was made in [9] where the topological string theory is based on harmonic maps. Very recently [10] a modified topological string theory was introduced. In that formulation a new interpretation of the $\Omega^{-1}$ points was given. Moreover, many aspects of the large $N$ limit of the $S U(N) Y M_{2}$ theory were reproduced in the zero coupling limit.

Another idea concerning the string action [2] was that it would be a NG action with an additional fold suppressing term. When we started this work, our hope was to convert the full NG action into a target space action that has two parts, a $Y M_{2}$ term and an action of interacting particles. In that case we might be able to add additional terms to cancel the contribution of the folds. This would provide the desired $Y M_{2}$ string action. Alternatively, a world sheet theory describing the folds could be amended so that the contribution of folds to the generating functional would have been cancelled. At present, since we do not have a full understanding of folds in neither of those two pictures, we cannot use our approach to get information pertaining to the string action.

Another model that may shed light on this problem is the one dimensional analogous model. In the space of maps from a world-line to a one dimensional target space, singular points are the analogs of the line singularities in the 2D case. The induced measure, the resulting target space quantum mechanical theory and additional related topics are under current investigation.

The construction of the stringy action of $Y M_{2}$ and $Q C D_{2}$ is still a challenging and important problem. 
As was explained in section (2) the contribution of maps with singular points are characterized by a set of different parameters associated with the various types of singularities. Thus, it is implied that the NG action is not one string model but it corresponds to a family of string models. In the stringy YM theory one is forced to set those parameters to be equal. It might be that the parameters are determined by the requirements of unitarity of the string theory and by the requirement that under a renormalization group flow to the infra-red the parameters remain relevant. In fact there is such an ambiguity in any infinite dimensional measure. In $\prod_{k=1}^{n} d x_{k}$ with metric $\sum_{k} d x_{k}^{2}$ we can rescale the measure by $\lambda$ and this would lead to rescaling of the metric by $\lambda^{2 / n}$. When $n \rightarrow \infty$ we can rescale the measure by a finite amount without changing the metric at all! It is interesting to wonder whether such a type of "rescaling" can remove the crumpling phase transition of the $D=2$ string. Namely, that the crumpling transition happens when maps with folds get counted with a weight that goes to infinity when the cut off goes to infinity. This is natural from the point of view of lattice regularization since the folds can pass anywhere on cell boundaries, and the number of maps with folds is proportional to the number of paths through cell boundaries which goes to infinity as the lattice constant goes to zero. However, when we damp the weight of the folds and make it finite (a finite measure of random walks in the target space) by adjusting the relative coefficient between the measure of maps with folds and maps without folds, maybe we can get rid of the crumpling transition.

Renormalizability and unitarity are two very important questions that were not addressed in our work. Even though we do not encounter divergences in the computations that we have performed. It is plausible that the contributions from infinitely small folds do diverge. In this case renormalizability arguments probably force us to include additional special points like "spiral-points". In section 4 we have hinted about the possibility that due to this type of renormalizability configurations with branch points "close" to folds may have a significant weight. A detailed analysis of these issues is under current investigation.

In the present work only maps from compact world sheets were considered. We have thus analyzed folds only in the framework of closed string theory. A discussion of folds in open strings both in the classical and semiclassical was presented in [4]. It was found that the string normal modes correspond to a completely folded strings having equal lengths that are os- 
cillating in that form. The exact interpretation of those folds in terms of the $Q C D_{2}$ picture is still unclear. The relations between that canonical analysis and our path-integral formulation has to be further investigated.

Another direction that has to be further explored is the space of maps to a higher genus target space. It seems that the latter is much more complex then the space of maps to the sphere.

As is evident from the present work the summation over folds is difficult. It may be advantageous to try and sum over specific families of folds. This was done in section (5), where the simple case of a non-self-overlapping string was investigated. In this case the summation problem was mapped into a lattice model which turned out to be the Baxter-Wu three-spin model in an external magnetic field. It would be important to find other lattice or continuum models which sum more complicated families of folds. This direction is under current investigation. A word of caution; even if we manage to sum over certain families of folds, there is apriori no guarantee that this partial sum will capture the physics of the full problem.

While completing this work we received two manuscripts that discuss related topics 16.

\section{Acknowledgment}

We would like to thank P. Di. Francesco, D. Kutasov, H. Neuberger and N. Seiberg for useful conversations. We would especially like to thanks W. Taylor and A. Zamolodchikov for helpful discussions and for their enlightening comments on various parts of this work. 


\section{Appendix A : Calculation of the fold matrix}

We will calculate the fold matrix for a fold that separates a region of covernumbers $(n, m)$ from a region of cover-numbers $(n+1, m+1)$. We will assume that there is no branch point exactly on the fold. This is certainly true in the "semi-classical" case as explained previously. However, we will consider spiral points (as in Figs.7,9) as well.

To check whether a specific point $P$ on a fold is a branch point or not, we have to surround the point by a small loop $\beta$ in the target-space. Each point in the loop corresponds to more than one point on the world-sheet if the number of covers at the vicinity of $P$ is greater than one. Next, we have to traverse the loop in the world-sheet, that is, pick a starting point on the world-sheet and go along $\beta$ on the corresponding sheets. In our route we will automatically switch sheets, and when we run into a fold we will have to reverse direction and return backwards in $\beta$ but on a reversely oriented sheet. Eventually we will return to our starting point on the same sheet. Counting our total winding number will give us the order of the branch point. If it is greater than 1 then $P$ is a branch-point, if the winding number is 1 then the point is a simple singular point on the fold, and if the winding number is zero then the point is inside a fold.

An example for the case of the smashed handle of Fig.3 will explain this procedure. We check the point $\mathrm{P}$ which is the common endpoint of $\gamma_{1}$ and $\gamma_{2}$ of (Fig.3). To the right of $\gamma_{1}, \gamma_{2}$ there is only one sheet which we denote ' $d$ '. To the left of $\gamma_{1}, \gamma_{2}$ there are three sheets, two of them $(a, c)$ with the same orientation as ' $d$ ' and the third $(b)$ with the opposite orientation. At the vicinity of $\gamma_{1}$ ' $d$ ' continues to ' $c$ ' and ' $a$ ' is joined to ' $b$ ' by a fold, whereas near $\gamma_{2}$ ' $d$ ' continues to ' $a$ ' and ' $c$ ' is joined to ' $b$ ' by a fold. The loop $\beta$ begins on ' $d$ ', when it passes over $\gamma_{2}$ it continues on ' $a$ '. $\beta$ changes direction when it meets $\gamma_{1}$ and returns on ' $b$ '. On $\gamma_{2}$ there is a second change of direction after which $\beta$ continues on ' $c$ ' and at last, it returns to the starting point when ' $c$ ' meets ' $d$ ' under $\gamma_{1}$. The total winding number is 1 so that, as expected, this is no branch-point.

We turn to the more general case. Assume there are $n$ sheets of orientation +1 and $m$ sheets of orientation -1 to the right of $\gamma_{1}, \gamma_{2}$, and there is one sheet more of each orientation to the left of $\gamma_{1}, \gamma_{2}$. Denote the sheets to the right as $\left\{a_{i}\right\}_{i=1}^{n}$ and $\left\{b_{j}\right\}_{j=1}^{m}$ and the sheets to the left as $\left\{c_{i}\right\}_{i=1}^{n+1}$ and $\left\{d_{j}\right\}_{j=1}^{m+1}$. We will first check the case that the folds $\gamma_{1}$ and $\gamma_{2}$ do not have a sheet in 
common. Then, we can label the sheets so that along $\gamma_{1}-c_{1}$ and $d_{1}$ are joined by the fold and so that $b_{j}$ is the continuation of $d_{j+1}$ and $a_{i}$ is the continuation of $c_{i+1}$. Along $\gamma_{2}$ we assume that $c_{n+1}$ and $d_{m+1}$ are joined by the fold, and that $a_{i}$ is the continuation of $c_{\sigma(i)}$ for some $\sigma \in S_{n}$ and likewise $b_{j}$ is the continuation of $d_{\tau(j)}$ for some $\tau \in S_{m}$.

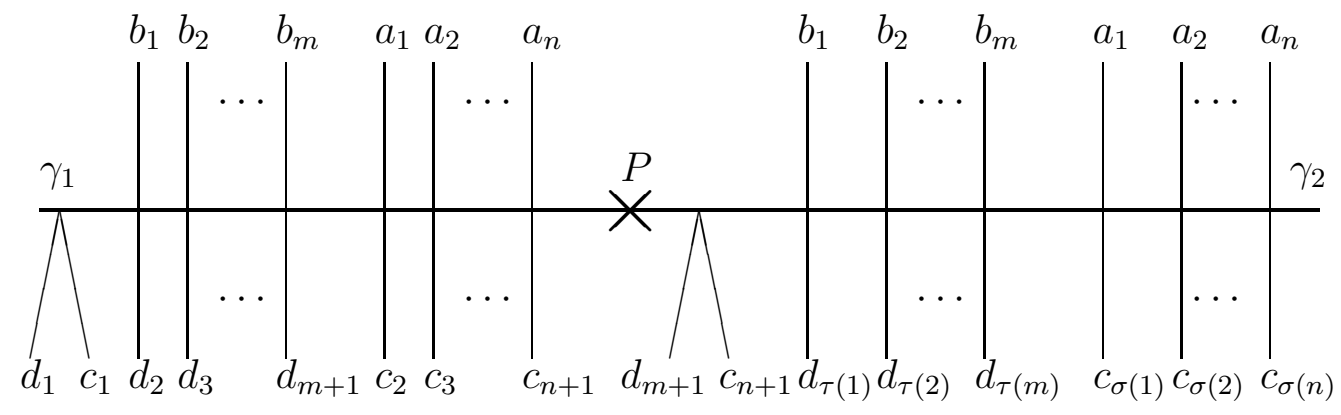

Fig. 15: Gluing of the sheets on two sides of a singular point $P$ on a fold. The fold does not have a sheet in common for both sides of $P$.

Start now with $a_{i}$ and make $r_{1}$ windings until either

$$
\sigma(\ldots(\sigma(\sigma(i)-1)-1) \ldots)-1=1
$$

in which case we have a winding of $r_{1}$ and we finish, or else:

$$
\sigma(\ldots(\sigma(\sigma(i)-1)-1) \ldots)=1
$$

then we change to $d_{1}$ and make $r_{2}$ anti-windings until

$$
\tau^{-1}\left(\ldots\left(\tau^{-1}\left(\tau^{-1}(1)+1\right)+1\right) \ldots\right)+1=m+1
$$


then, we switch to $c_{n+1}$ and next to $a_{n}$ and make $r_{3}$ more windings until:

$$
\sigma(\ldots(\sigma(\sigma(n)-1)-1) \ldots)-1=i
$$

(we have to reach $a_{i}$ before we reach $c_{1}$ in order not to get trapped in circles on a trajectory that does not contain $a_{i} !$ )

So, to summarize, there are three possibilities of trajectories:

1. trajectories of the form

$$
a_{t_{1}} \rightarrow c_{\sigma\left(t_{1}\right)} \rightarrow a_{t_{2}} \rightarrow c_{\sigma\left(t_{2}\right)} \rightarrow \ldots \rightarrow a_{t_{r}}
$$

with

$$
\begin{aligned}
t_{i+1} & =\sigma\left(t_{i}\right)-1, \quad i=1, \ldots r-1 \\
t_{r} & =t_{1}
\end{aligned}
$$

the winding number is $r-1$ so in order not to have a branch point we require that this will not be possible for $r>2$.

2. trajectories of the form

$$
b_{s_{1}} \rightarrow d_{\tau\left(s_{1}\right)} \rightarrow b_{s_{2}} \rightarrow d_{\tau\left(s_{2}\right)} \rightarrow \ldots \rightarrow b_{s_{r}}
$$

with

$$
\begin{aligned}
s_{i+1} & =\tau\left(s_{i}\right)-1, \quad i=1, \ldots r-1 \\
s_{r} & =s_{1}
\end{aligned}
$$

the winding number is again $r-1$ so in order not to have a branch point we require that this will not be possible for $r>2$.

3. trajectories of the form

$$
\begin{aligned}
d_{1} & =d_{s_{1}} \rightarrow b_{\tau^{-1}\left(s_{1}\right)} \rightarrow d_{s_{2}} \rightarrow b_{\tau^{-1}\left(s_{2}\right)} \rightarrow \ldots \rightarrow d_{s_{r}} \\
& =d_{m+1} \rightarrow c_{n+1} \rightarrow a_{n} \\
& =a_{t_{1}} \rightarrow c_{\sigma\left(t_{1}\right)} \rightarrow \ldots \rightarrow c_{\sigma\left(t_{p}\right)}=c_{1}
\end{aligned}
$$

with

$$
s_{1}=1
$$




$$
\begin{aligned}
s_{i+1} & =\tau^{-1}\left(s_{i}\right)+1, \quad i=1, \ldots, r-1 \\
s_{r} & =m+1 \\
t_{1} & =n \\
t_{i+1} & =\sigma\left(t_{i}\right)-1, \quad i=1, \ldots, p-1 \\
\sigma\left(t_{p}\right) & =1
\end{aligned}
$$

The winding number is $r-p-1$ so in order to have no branch points we require that this will not be possible for $|r-p-1|>2$ In fact, for $r-p=1$ this construction describes a spiral point of order $r$ as in Fig.9. For $r-p=2$ We get a spiral point of order $r-\frac{1}{2}$ as in Fig.7.

Next, for the case that the folds do have a sheet in common, we can label the sheets so that along $\gamma_{1}-c_{n+1}$ and $d_{1}$ are joined by the fold and that $b_{j}$ is the continuation of $d_{j+1}$ and $a_{i}$ is the continuation of $c_{i}$. Along $\gamma_{2}$ we assume that $c_{n+1}$ and $d_{m+1}$ are joined by the fold, and that $a_{i}$ is the continuation of $c_{\sigma(i)}$ for some $\sigma \in S_{n}$ and likewise $b_{j}$ is the continuation of $d_{\tau(j)}$ for some $\tau \in S_{m}$. 


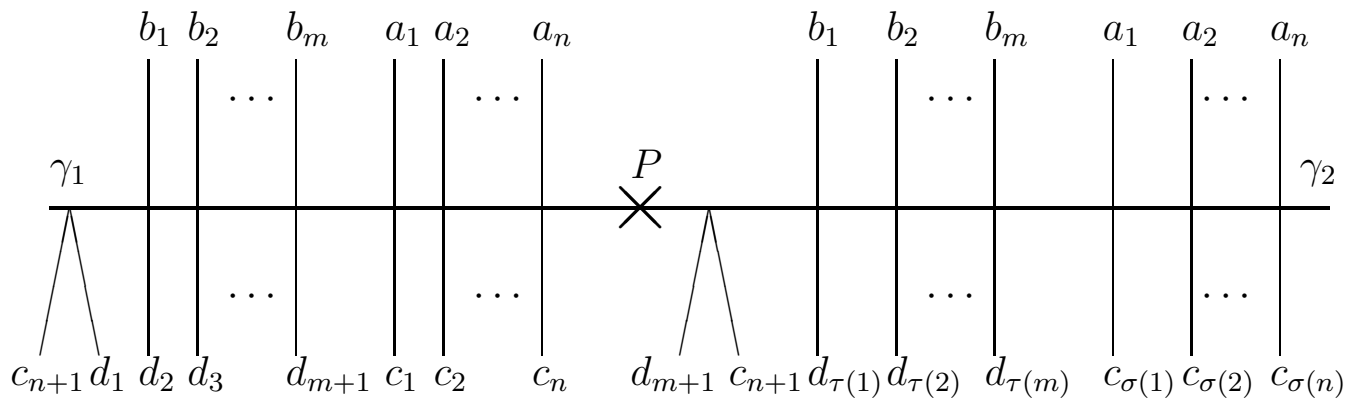

Fig. 16: Gluing of the sheets on two sides of a singular point $P$ on a fold. The fold has a sheet in common for both sides of $P$.

We have the following restrictions:

1. from trajectories of the form

$$
a_{t_{1}} \rightarrow c_{\sigma\left(t_{1}\right)} \rightarrow a_{t_{2}} \rightarrow c_{\sigma\left(t_{2}\right)} \rightarrow \ldots \rightarrow a_{t_{r}}
$$

with

$$
\begin{aligned}
t_{i+1} & =\sigma\left(t_{i}\right), \quad i=1, \ldots r-1 \\
t_{r} & =t_{1}
\end{aligned}
$$

the winding number is $r-1$ so in order to have no branch points we require that this will not be possible for $r>2$

2. trajectories of the form

$$
b_{s_{1}} \rightarrow d_{\tau\left(s_{1}\right)} \rightarrow b_{s_{2}} \rightarrow d_{\tau\left(s_{2}\right)} \rightarrow \ldots \rightarrow b_{s_{r}}
$$


with

$$
\begin{aligned}
s_{i+1} & =\tau\left(s_{i}\right)-1, \quad i=1, \ldots r-1 \\
s_{r} & =s_{1}
\end{aligned}
$$

the winding number is again $r-1$ so in order to have no branch points we require that this will not be possible for $r>2$

3. trajectories of the form

$$
\begin{aligned}
d_{1} & =d_{t_{1}} \rightarrow b_{\tau^{-1}\left(t_{1}\right)} \rightarrow d_{t_{2}} \rightarrow b_{\tau^{-1}\left(t_{2}\right)} \rightarrow \ldots \rightarrow d_{t_{r}} \\
& =d_{m+1} \rightarrow c_{n+1} \rightarrow d_{1}
\end{aligned}
$$

with

$$
\begin{aligned}
t_{1} & =1 \\
t_{i+1} & =\tau^{-1}\left(t_{i}\right)+1, \quad i=1, \ldots, r-1 \\
t_{r} & =m+1
\end{aligned}
$$

the winding number is $r-1$ so in order to have no branch points we require that this will not be possible for $r>2$

The first two cases correspond to a branch point that is on a "spectator" sheets (i.e. disjoint from the sheets of the fold in a small neighborhood of $P$ ). The third case corresponds to a branch point that "sits" on the fold itself and can be described as a limiting case of a branch point that approaches a simple singular point on a fold.

Returning to the fold matrix $F_{\sigma_{2} \tau_{2}}^{\sigma_{1} \tau_{1}}$, it can be seen, from all that has been said above, that for a spiral point of the type of Fig.9 and of order $k, \sigma_{2}$ and $\tau_{2}$ can be written in terms of $\sigma_{1}$ and $\tau_{1}$ as:

$$
\begin{aligned}
\sigma_{2} & =\sigma_{1} \circ\left((n+1) z_{1} z_{2} \ldots z_{k}\right) \\
\tau_{2} & =\tau_{1} \circ\left((m+1) w_{1} w_{2} \ldots w_{k}\right)
\end{aligned}
$$

where $\left(a_{1} a_{2} \ldots a_{r}\right)$ denotes a cyclic permutation in which $a_{1}$ goes to $a_{2}, a_{2}$ goes to $a_{3}$ and so on, and $z_{1}, z_{2}, \ldots, z_{k}, w_{1}, w_{2}, \ldots, w_{k}$ are some sheet numbers.

For a spiral point of the type of Fig.7 we get:

$$
\begin{aligned}
\sigma_{2} & =\sigma_{1} \circ\left((n+1) z_{1} z_{2} \ldots z_{k-1}\right) \\
\tau_{2} & =\tau_{1} \circ\left((m+1) w_{1} w_{2} \ldots w_{k}\right)
\end{aligned}
$$


or

$$
\begin{aligned}
\sigma_{2} & =\sigma_{1} \circ\left((n+1) z_{1} z_{2} \ldots z_{k}\right) \\
\tau_{2} & =\tau_{1} \circ\left((m+1) w_{1} w_{2} \ldots w_{k-1}\right)
\end{aligned}
$$

If we include the "spiral coupling constant" $\rho_{s p}$ for points of the type of Fig.9 and $\rho_{s p}^{\prime}$ for points of the type of Fig.7, we get:

$$
\begin{aligned}
F_{\sigma_{2} \tau_{2}}^{\sigma_{1} \tau_{1}}= & \sum_{k=1}^{\infty} \#\left\{z_{1}, z_{2}, \ldots, z_{k}, w_{1}, w_{2}, \ldots, w_{k} \mid\right. \\
& \sigma_{2}=\sigma_{1} \circ\left((n+1) z_{1} z_{2} \ldots z_{k}\right), \\
& \tau_{2}=\tau_{1} \circ\left((m+1) w_{1} w_{2} \ldots w_{k}\right\} \rho_{s p}^{k} \\
+ & \sum_{k=0}^{\infty} \#\left\{z_{1}, z_{2}, \ldots, z_{k-1}, w_{1}, w_{2}, \ldots, w_{k} \mid\right. \\
& \sigma_{2}=\sigma_{1} \circ\left((n+1) z_{1} z_{2} \ldots z_{k-1}\right), \\
& \tau_{2}=\tau_{1} \circ\left((m+1) w_{1} w_{2} \ldots w_{k}\right\} \rho_{s p}^{\prime k} \\
+ & \sum_{k=0}^{\infty} \#\left\{z_{1}, z_{2}, \ldots, z_{k}, w_{1}, w_{2}, \ldots, w_{k-1} \mid\right. \\
& \sigma_{2}=\sigma_{1} \circ\left((n+1) z_{1} z_{2} \ldots z_{k}\right), \\
& \tau_{2}=\tau_{1} \circ\left((m+1) w_{1} w_{2} \ldots w_{k-1}\right\} \rho_{s p}^{\prime}{ }^{k}
\end{aligned}
$$

The value $k=0$ was included in the last two terms. This is the contribution of the "simple singular points" on the fold.

\section{Appendix B : Calculation of the eigenvalues of the fold matrix}

We will first calculate the eigenvalues of the fold-matrix in the case $m=0$. We will start with the non-restricted fold matrix (which is the restricted fold matrix $\left.F_{(\phi=1, \psi=1)}\right)$.

For $m=0$, the folds always have a common sheet, say $d_{1}$. We will assume that at the fold $\gamma_{i}-d_{1}$ is attached to $c_{\alpha_{i}}$ with $1 \leq \alpha_{i} \leq n+1$ ( $i$ being the index of the fold arc). We will also assume that $a_{j}$ is joined to $c_{\sigma_{i}(j)}$ with $\sigma_{i}:\{1, \ldots, n\} \rightarrow\{1, \ldots, n+1\}$. From the previous chapter (when we set $\rho_{s p}$ 
and $\rho_{s p}^{\prime}$ to zero in 64, since there are no spiral points with $k>0$ for $m=0$, and $\tau$ can be dropped as well when $m=0$.) we see that $\sigma_{i}$ must satisfy

$$
\begin{aligned}
\sigma_{i}(x) & =\sigma_{i+1}(x) \text { for } x \notin\left(\sigma_{i}^{-1}\left(\alpha_{i}\right), \sigma_{i+1}^{-1}\left(\alpha_{i+1}\right)\right) \\
\sigma_{i}\left(\sigma_{i+1}^{-1}\left(\alpha_{i+1}\right)\right) & =\alpha_{i} \\
\sigma_{i+1}\left(\sigma_{i}^{-1}\left(\alpha_{i}\right)\right) & =\alpha_{i+1}
\end{aligned}
$$

and we get:

$$
F_{\sigma_{2}}^{\sigma_{1}}=\sum_{y=1}^{n} \delta\left(\sigma_{2}^{-1} \sigma_{1}((n+1) y)\right)=D_{R e g}\left(\sum_{y=1}^{n}((n+1) y)\right.
$$

In the sum $\sum_{y}$ only $y=\sigma_{1}^{-1} \sigma_{2}(n+1)$ can give a nonzero $\delta$-function, and $D_{\text {reg }}(\sigma)$ is the matrix of the permutation $\sigma \in S_{n+1}$ in the regular $(n+1) ! \times$ $(n+1)$ ! representation.

The regular representation of $S_{k}$ can be decomposed into irreducible representations. It is best to use the Yamanouchi formalism [17], in which case the representations are labeled by the value of the sums of the permutations in all equivalence classes of $S_{k}$, and to those are added the sums of the permutations in all equivalence classes of $S_{r}$ for $r=1, \ldots, k-1$, thus obtaining a maximal commuting set of operators. In this representation, for $S_{n+1}$ :

$$
\begin{aligned}
\sum_{r=1}^{n}(r,(n+1)) & =\frac{1}{2} \sum_{1 \leq x, y \leq(n+1)}(x y)-\frac{1}{2} \sum_{1 \leq x, y \leq n}(x y) \\
& =\lambda_{(2)}^{(v)}-\lambda_{(2)}^{\left(v^{\prime}\right)} \\
& =\frac{1}{2}+\frac{1}{2} \sum_{l=1}^{q}\left(v_{l}\left(v_{l}-2 l\right)-v_{l}^{\prime}\left(v_{l}^{\prime}-2 l\right)\right) \\
& =\frac{1}{2}+\frac{1}{2}\left(v_{t}\left(v_{t}-2 l\right)-\left(v_{t}-1\right)\left(v_{t}-1-2 l\right)\right)=v_{t}-t
\end{aligned}
$$

Where $v$ is a partition $n+1=\sum_{l=1}^{q} v_{l}$ which represents a Young diagram for $S_{n+1}$ and $v^{\prime}$ is a Young diagram for $S_{n}$ that is obtained from $v$ by taking off one square in an allowed way (that is, leaving $v_{1}^{\prime} \geq v_{2}^{\prime} \geq \ldots \geq v_{l}^{\prime}$ ) which we denote by $v_{t}^{\prime}=v_{t}-1 . \lambda_{(2)}^{(v)}$ is the character for 2-cycles in $(v)$, that is the scalar that represents $\frac{1}{2} \sum_{1 \leq x, y \leq(n+1)}(x y)$ in the $(v)$ representation. Likewise $\lambda_{(2)}^{\left(v^{\prime}\right)}$ represents $\frac{1}{2} \sum_{1 \leq x, y \leq n}(x y)$ in the $\left(v^{\prime}\right)$ representation. 
We see that the eigenvalues turn out to be $\left(v_{t}-t\right)$, and they come in pairs of positive-negative eigenvalues. The eigenvalue of $\left(t-v_{t}\right)$ corresponds to a Young tableau that is the mirror of the first Young tableau.

\section{The Restricted Fold Matrices}

To calculate (28) we need the eigenvalues of all of the restricted fold matrices $F_{\left(\phi \in S_{n+1}, \psi \in S_{n}\right)}$ (we omit $\theta, \beta$ since they are trivial for $m=0$ ).

In the following equations we consider $\psi \in S_{n} \subset S_{n+1}$.

For given $\phi \in S_{n+1}$ and $\psi \in S_{n}$ we are interested in the $\sigma_{i}$-s that satisfy

$$
\sigma_{i}^{-1} \phi \sigma_{i}=\psi
$$

(see (27)) .

This means that unless $\psi$ and $\phi$ are of the same equivalence class the restricted fold matrix is identically zero. So suppose that

$$
\psi=\rho^{-1} \phi \rho
$$

so that we are interested only in $\sigma_{i}=\rho \theta_{i}$ where $\theta_{i} \in S_{n+1}$ is a permutation that commutes with $\psi$. From (66) and (27) we obtain (recall that $(x, y)$ is the transposition permutation that switches $x$ with $y)$ :

$$
\begin{aligned}
\left(F_{(\psi, \phi)}\right)_{\theta_{2}}^{\theta_{1}} & =\delta\left(\sigma_{2}^{-1} \circ \sigma_{1} \circ\left((n+1) \sigma_{2}(n+1)\right)\right) \\
& =\delta\left(\sigma_{2}^{-1} \circ\left(\sigma_{1}(n+1) \sigma_{2}(n+1)\right) \circ \sigma_{1}\right) \\
& =\delta\left(\theta_{2}^{-1} \rho^{-1} \circ\left(\sigma_{1}(n+1) \sigma_{2}(n+1)\right) \circ \rho \theta_{1}\right) \\
& =\delta\left(\theta_{2}^{-1} \circ\left(\theta_{1}(n+1) \theta_{2}(n+1)\right) \circ \theta_{1}\right) \\
& =\left(F_{(1,1)}\right)_{\theta_{2}}^{\theta_{1}}
\end{aligned}
$$

We have changed the indices of $F$ to $\theta_{1}, \theta_{2}$ since the $\sigma_{i}$ that come into consideration are in one to one correspondence with the $\theta_{i}$-s.

This means that $F$ can be put in block form as follows:

The equivalence class of $\psi$ is in one to one correspondence with the partitions of $n$ since

$\psi=\left(a_{1}\right)\left(a_{2}\right) \ldots\left(a_{r_{1}}\right)\left(b_{1} c_{1}\right)\left(b_{2} c_{2}\right) \ldots\left(b_{s_{1}} c_{t_{1}}\right) \ldots(3-$ cycles $) \ldots(4-$ cycles $) \ldots$ 
such that $(n+1)$ is included in the 1 -cycles. The permutations $\tau$ that commute with $\psi$ are those that transform the indices of the cycles into other indices that describe the same cycle. This means, in particular, that if $\left(a_{i 1}, a_{i 2}, \ldots, a_{i k}\right)$ are all the $k-$ cycles with $i=1, \ldots, N_{k}$, then $\tau\left(a_{i j}\right)=a_{i^{\prime} j^{\prime}}$ for some $i^{\prime} j^{\prime}$ and if $\tau\left(a_{i j}\right)=a_{i^{\prime} j^{\prime}}$ then $\left.\tau\left(a_{i r}\right)=a_{i^{\prime}\left(j^{\prime}+r-j\right.}(\bmod k)\right)$ so as to transform the $i$-th $k$-cycle to the $i^{\prime}$-th $k$-cycle.

What pairs of $\theta_{1}, \theta_{2}$ that commute with $\psi$ give a non-zero restricted fold matrix element? According to (70) $\theta_{1}$ and $\theta_{2}$ must differ just by switching two elements which are $\theta_{1}(n+1)$ and $\theta_{2}(n+1)$. But, since they commute with $\psi$, $\theta_{1}(n+1)$ and $\theta_{2}(n+1)$ must be 1-cycles of $\psi$. Let $N_{k}(\psi)$ be the number of $k$ cycles of $\psi$. Thus, the restricted fold matrix is equal to $\prod_{k=2}^{n}\left(N_{k}(\psi)^{k} N_{k}(\psi)\right.$ !) copies of the (non-restricted) $N_{1}(\psi) ! \times N_{1}(\psi)$ ! fold matrix. This is because each pair $\theta_{1}, \theta_{2}$ that gives a nonzero matrix element in $(70)$ can be written as

$$
\begin{aligned}
& \theta_{1}=\theta_{1}^{\prime} \circ \theta_{1}^{\prime \prime} \\
& \theta_{2}=\theta_{2}^{\prime} \circ \theta_{2}^{\prime \prime}
\end{aligned}
$$

where $\theta_{1}^{\prime}, \theta_{2}^{\prime}$ are permutations of the $N_{1}(\psi) 1$-cycles of $\psi$ and $\theta_{1}^{\prime \prime}, \theta_{2}^{\prime \prime}$ are permutations among the $k>1$ cycles. according to what was said above $\theta_{1}^{\prime \prime}=\theta_{2}^{\prime \prime}$ in order to give a non-zero matrix element. The number $\prod_{k=2}^{n}\left(N_{k}(\psi)^{k} N_{k}(\psi)\right.$ !) is the number of possible values of $\theta_{1}^{\prime \prime}$.

\section{A General $(n, m)$ Region}

If we do not allow "spiral" singular points, that is, put $\rho_{s p}=0$ in (64), we get a fold matrix that at each singular point leaves one of the covers that joins a fold unchanged.

That is, the matrix elements $F_{\sigma_{2} \tau_{2}}^{\sigma_{1} \tau_{1}}$ are sums of two terms, one is proportional to $\delta\left(\tau_{1}^{-1} \tau_{2}\right)$, and the other is proportional to $\delta\left(\sigma_{1}^{-1} \sigma_{2}\right)$. In this case we can write $F(n, m)$ as a sum of tensor products:

$$
F(n, m)=F(n, 0) \otimes 1+1 \otimes F(0, m)
$$

where 1 is the identity matrix. The eigenvalues of $F(n, m)$ are the sums of eigenvalues of $F(n, 0)$ and $F(0, m)$. 


\section{References}

[1] D. J. Gross, "Some New/Old approaches to QCD", PUPT-1355, Presented at Mtg. on Integrable Quantum Field Theories, Como, Italy, Sep 14-19, 1992, and at Int. Workshop on String Theory, Quantum Gravity and the Unification of Fundamental Interactions, Rome, Italy, 21-26 Sep 1992. Published in Rome String Theory Wkshp.1992:251 -268 (QCD161:I409: 1992) also in Como ARW QFT 1992:317-330 (QC174.45:N2:1992);

"Two Dimensional QCD as a String Theory", Nucl. Phys. B400 (1993) 161.

[2] D. J. Gross and W. Taylor, Nucl. Phys. B400,(1993)181-210.

[3] D. J. Gross and W. Taylor, "Twists And Wilson Loops In The String Theory Of QCD", Nucl. Phys. B403 (1993) 395.

[4] W.A. Bardeen, I. Bars, A. Hanson and R.Peccei, Phys. Rev. D13 (1976) 1744;Phys. Rev. D13 (1976) 2193.

I. Bars, $Q C D$ and Strings in 2D, USC-93/HEP-B3.

[5] J. A. Minahan, Phys. Rev. D47 (1993) 3430.

[6] J. A. Minahan, and A. P. Polychronakos, Phys. Lett. 312B (1993) 155.

M. R. Douglas, "Some comments on QCD string”, , RU-94-09, Jan 1994, hep-th@xxx.lanl.gov - 9401073.

M. R. Douglas, "Conformal field theory techniques in large N YM theory", HEPTH-9311130, May 1993. Presented at Cargese Workshop on Strings, Conformal Models and Topological Field Theories, Cargese, France, May 12-26, 1993; hep-th@xxx.lanl.gov - 9311130.

S. G. Naculich, Harold A. Riggs, Howard J. Schnitzer Phys. Lett. 319B (1993) 466.

S. Ramgoolam, "Comment on two dimensional $O(N)$ and $S P(N)$ YangMills theories as string theories", YCTP-P16-93, hep-th 930785

J. Baez, W. Taylor (MIT, LNS), "Strings and two-dimensional QCD for finite N", MIT-CTP-2266, Jan 1994. 19pp hep-th@xxx.lanl.gov - 9401041.

[7] A. Polyakov, Nucl. Phys. B40 (1982) 235.

[8] M. Bershadsky, S. Cecotti, H. Ooguri, C. Vafa, Nucl. Phys. B405 (1993) 279 . 
[9] P. Horava, "Topological String and QCD in Two Dimensions", hep-th/ 9311156 EFI-93-66.

[10] S. Cordes, G. Moore and S. Ramgoolam, "Large $N$ 2D Yang-Mills Theory and Topological String Theory", hepth-9402107, YCTP-P23-93.

[11] A. Strominger, Phys. Lett. 101B (1986) 271.

[12] Baxter, R. J. and Wu, F. Y. Phys. Rev. Lett. 31 (1973) 1294.

[13] Baxter, R. J., "Exactly Solved Models in Statistical Mechanics", Academic Press.

[14] V. A. Kazakov, Nucl. Phys. B179 (1981) 283

[15] E. Witten, Comm. Math. Phys. 118 (1988) 411.

[16] J. Pawelczyk, "Toward QCD String: No folds", (Warsaw U., ITP), IFT1-94, Jan 1994, hep-th 9403175.

P. Di Francesco and E. Guitter, "Entropy of Folding of the Triangular Lattice", Saclay preprint T/94018 cond-mat/9402058.

[17] For Example : Jin-Quan Chen, "Group Representation Theory For Physicists", World Scientific 1989 